\title{
APLICACIÓN DE LA METODOLOGÍA DE ANÁLISIS DE CICLO DE VIDA PARA EVALUAR EL DESEMPEÑO AMBIENTAL DE SISTEMAS DE GESTIÓN DE RESIDUOS EN IBEROAMERICA
}

\author{
María D. BOVEA ${ }^{1 *}$, Samantha E. CRUZ-SOTELO ${ }^{2}$, Irma MERCANTE ${ }^{3}$, \\ Claudia COUTINHO-NÓBREGA ${ }^{4}$, Mónica ELJAIEK-URZOLA ${ }^{5}$ y Valeria IBÁÑEZ-FORÉS ${ }^{1}$
}

\author{
${ }^{1}$ Departamento de Ingeniería Mecánica y Construcción, Universitat Jaume I, Castellón, España \\ ${ }^{2}$ Facultad de Ingeniería, Universidad Autónoma de Baja California, Mexicali, México \\ ${ }^{3}$ CEIRS, Facultad de Ingeniería. Universidad Nacional de Cuyo, Mendoza, Argentina \\ ${ }^{4}$ Departamento de Engenharia Civil e Ambiental, Universida de Federal da Paraíba, Joao Pessoa, Brasil \\ ${ }^{5}$ Facultad de Ingeniería, Campus Piedra de Bolívar, Universidad de Cartagena, Cartagena, Colombia \\ *Autor para correspondencia: bovea@uji.es
}

(Recibido noviembre 2014; aceptado diciembre 2015)

Palabras clave: ACV, residuos, caso de aplicación

\section{RESUMEN}

La metodología de Análisis de Ciclo de Vida es la técnica más adecuada para evaluar el desempeño medioambiental de los sistemas de gestión de residuos sólidos. Durante los últimos años, se ha ido extendiendo su implementación en diferentes países iberoamericanos. Así pues, este artículo presenta cinco experiencias de aplicación de la metodología de Análisis de Ciclo de Vida para evaluar el comportamiento ambiental de: un sistema de recogida selectiva de residuos domiciliarios en España; un sistema de gestión de residuos de aparatos eléctricos y electrónicos y específicamente terminales de telefonía móvil en México; un sistema de gestión de residuos de construcción y demolición en Argentina; la incorporación de recogida selectiva en un sistema de gestión de residuos domiciliarios en Brasil; y finalmente, un sistema de gestión de residuos domiciliarios en Colombia. Para cada uno de los casos de aplicación se ha conformado un inventario, identificándose como principal hándicap, la falta de datos de inventario en bases de datos comerciales adaptados a diferentes países, a diferentes fracciones y a diferentes tratamientos de gestión de residuos. Para ello, para modelar los inventarios se ha realizado una combinación de datos primarios y secundarios. Finalmente, se ha obtenido la contribución al impacto de las diferentes etapas que conforman el ciclo de vida de cada sistema de gestión de residuos, sirviendo los resultados obtenidos como punto de partida para la selección e implantación de medidas que favorezcan la mejora ambiental de los sistemas de gestión de residuos.

Key words: LCA, waste, case study

\begin{abstract}
The Life Cycle Assessment methodology is the most appropriate technique for evaluating municipal solid waste management systems. In recent times, its implementation has been spreading in different Latin American countries. So, this paper presents five applications of the Life Cycle Assessment methodology with the aim of evaluating the environmental performance of: a selective collection system for household waste
\end{abstract}


in Spain; a waste electrical and electronic equipment management system, mainly focused on mobile phone devices, in Mexico; a construction and demolition waste management system in Argentina; the incorporation of selective collection in a municipal solid waste management system in Brazil; and finally, a municipal solid waste management system for household in Colombia. For each case study it has been made an inventory, identifying as a main handicap, the lack of inventory data in commercial databases adapted to different countries, different factions and different treatments of waste management. To solve this problem, inventory modeling has been performed as a combination of primary and secondary data. Finally, the contribution to different impact categories of stage of each life cycle waste management system has been obtained. Results provide a starting point for the selection and implementation of measures to promote the environmental improvement of waste management systems.

\section{INTRODUCCIÓN}

La metodología de Análisis de Ciclo de Vida (ACV) se considera que es la mejor herramienta para evaluar el desempeño medioambiental de sistemas de gestión de residuos (Arena et al. 2003), ya que permite evaluar, desde una perspectiva global, todos los impactos ambientales que ocasiona la gestión integral de los residuos, facilitando la comparación entre distintas alternativas de tratamiento (Mc Dougall et al. 2001, Laurent et al. 2014).

La mayoría de los estudios que aplican la metodología ACV a la evaluación ambiental de los sistemas de gestión de residuos corresponden a casos de aplicación en países europeos, asiáticos o norteamericanos (Laurent et al.2014, Hou et al.2015). Sin embargo, su aplicación en algunos países iberoamericanos se encuentra todavía en un estado incipiente, tal y como se observa en el cuadro I. España, seguida de Brasil y Portugal, son los países con mayor número de publicaciones en este campo. Además, los residuos domiciliarios, tanto en general, como sus fracciones orgánicas, envases y papel-cartón, son los que mayormente han sido objeto de estudio, seguidos de los residuos de construcción y demolición y los industriales-agroalimentarios (Fig. 1).

Si se analiza la variable temporal, la figura 2 muestra la evolución del número de publicaciones por año y país; España presenta una evolución ascendente en el número de publicaciones desde el inicio de la década del 2000, mientras que en el resto de países analizados los estudios se iniciaron en la década del 2010.

Teniendo en cuenta este contexto, este artículo se centra en analizar la contribución que los países iberoamericanos están realizando a la investigación en el campo de aplicación de la metodología ACV a la gestión de los residuos, mediante la presentación de cinco casos de aplicación en cinco países: España, México, Argentina, Brasil y Colombia.

\section{MATERIALES Y MÉTODOS}

\section{Experiencias de aplicación del ACV a sistemas de gestión de residuos}

Esta sección presenta casos de aplicación de la metodología ACV para la evaluación ambiental de los sistemas de gestión de varios tipos de residuos, en los siguientes países iberoamericanos:

- España: sistema de recogida selectiva y gestión de residuos domiciliarios.

- México: gestión de residuos de aparatos eléctricos y electrónicos correspondientes a los terminales de telefonía móvil.

- Argentina: escenarios para la gestión de residuos de construcción y demolición.

- Brasil: sistema de recogida y gestión de residuos domiciliarios.

- Colombia: escenarios para la gestión de residuos domiciliarios.

Todos estos casos de estudio se han realizado siguiendo las recomendaciones de la norma ISO 14040-44 (2006) mediante la aplicación del software SimaPro (2013). Tras establecer cuáles son los límites del sistema y el alcance del estudio, y definir la unidad funcional a analizar en cada caso, se presenta el modelo de inventario utilizado en cada uno, diferenciando datos primarios (datos de campo recogidos de empresas gestoras de residuos) y secundarios (procedentes de bases de datos comerciales). La etapa de evaluación del impacto se realiza siguiendo los elementos obligatorios propuestos por la ISO 14040 (2006), es decir, obteniendo indicadores para diferentes categorías de 
CUADRO I. REVISIÓN DE PUBLICACIONES SOBRE APLICACIÓN DE LA METODOLOGÍA ACV A LA GESTIÓN DE RESIDUOS SÓLIDOS EN PAÍSES IBEROAMERICANOS

\begin{tabular}{|c|c|c|c|c|c|c|c|c|c|c|c|c|c|}
\hline \multirow[b]{2}{*}{ País } & \multirow[b]{2}{*}{ Referencia } & \multicolumn{12}{|c|}{ Tipo de residuo } \\
\hline & & 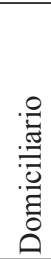 & 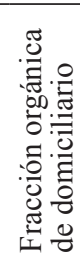 & 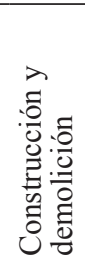 & 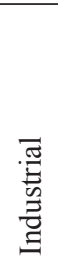 & 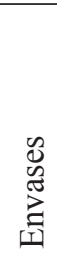 & 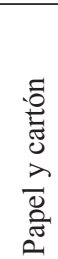 & 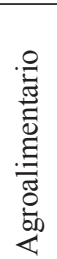 & $\begin{array}{l}\stackrel{0}{8} \\
\stackrel{0}{0} \\
\end{array}$ & 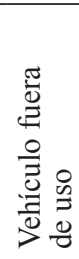 & ·怘 & 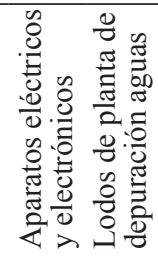 & $\frac{\pi}{\frac{\pi}{0}}$ \\
\hline \multirow{27}{*}{ España } & Ruiz et al. (2014) & & & & & & $x$ & & & & & & \\
\hline & Aranda et al. (2013) & $x$ & & & & & & & & & & & \\
\hline & Martínez et al. (2013) & & & $x$ & & & & & & & & & \\
\hline & Montejo et al. (2013) & $x$ & & & & & & & & & & & \\
\hline & Puig et al. (2013) & & & & $\times$ & & & & & & & & \\
\hline & Mercante et al. (2012) & & & $\times$ & & & & & & & & & \\
\hline & Fullana i Palmer et al. (2011) & & & & $\times$ & & & & & & & & \\
\hline & Uggetti et al. (2011) & & & & & & & & & & & $x$ & \\
\hline & Bovea et al. (2010) & $\times$ & & & & & & & & & & & \\
\hline & Colón et al. $(2010,2012)$ & & $\times$ & & & & & & & & & & \\
\hline & Margallo et al. (2010) & $x$ & & & & & & & & & & & \\
\hline & Meneses et al. (2010) & & & & $x^{1}$ & & & & & & & & \\
\hline & Muñoz et al. (2010) & & $x$ & & & & & & & & & & \\
\hline & Ortiz et al. (2010) & & & $x$ & & & & & & & & & \\
\hline & Ortiz-Rodríguez et al. (2010) & & & $x$ & & & & & & & & & \\
\hline & Rives et al. (2010) & $\times$ & & & & & & & & & & & \\
\hline & Cadena et al. (2009) & & $x$ & & & & & & & & & & \\
\hline & Iriarte et al. (2009) & $\times$ & & & & & & & & & & & \\
\hline & Ruggieri et al. (2009) & & & & & & & & & & & $x$ & \\
\hline & Bovea \& Powell (2006) & $\times$ & & & & & & & & & & & \\
\hline & Güereca et al. $(2006,2007)$ & & $x$ & & & & & & & & & & \\
\hline & Rivela et al. (2006) & & & & & & & & & & & & $\times$ \\
\hline & Hospido et al. $(2005,2010)$ & & & & & & & & & & & $\times$ & \\
\hline & Muñoz et al. (2004) & $x$ & & & & & & & & & & & \\
\hline & Rodríguez-Iglesias et al. (2003) & $\times$ & & & & & & & & & & & \\
\hline & Ciroth et al. $(2002 a, b)$ & $x$ & & & & & & & & & & & \\
\hline & Rieradevall et al. (1997) & $\times$ & & & & & & & & & & & \\
\hline \multirow{6}{*}{ Portugal } & Ferrão et al. (2014) & & & & & $x$ & & & & & & & \\
\hline & Fonseca et al. (2013) & & & & & & & & & $x$ & & & \\
\hline & González-García et al. (2013) & & & & & & & $x^{2}$ & & & & & \\
\hline & Pires \& Martinho (2013) & & & & & & & & $\times$ & & & & \\
\hline & Coelho \& de Brito, 2012) & & & $\times$ & & & & & & & & & \\
\hline & Pires et al. (2011) & $\times$ & & & & & & & & & & & \\
\hline \multirow{3}{*}{ Chile } & Bezama et al. (2013) & $\times$ & & & & & & & & & & & \\
\hline & James (2012) & & & & & & $x$ & & & & & & \\
\hline & Muñoz \& Navia (2011) & & & & $\times$ & & & & & & & & \\
\hline Colombia & Bojacá et al. (2014) & & & & & & & $x^{3}$ & & & & & \\
\hline México & Romero-Hernández et al. (2009) & & & & & $\times$ & & & & & & & \\
\hline \multirow{9}{*}{ Brasil } & Rubin et al. (2014) & & & & & & & & & & & $x$ & \\
\hline & Zanghelini et al. (2014) & & & & & & & & & & & $x$ & \\
\hline & Leme et al. (2014) & $\times$ & & & & & & & & & & & \\
\hline & Soares et al. (2013) & & & & & & & & & & $x$ & & \\
\hline & Vinyes et al. (2013) & & & & & & & & $\times$ & & & & \\
\hline & Leme et al. (2012) & $\times$ & & & & & & & & & & & \\
\hline & Frietas et al. (2012) & $x$ & & & & & & & & & & & \\
\hline & Stichnothe \& Azapagic (2009) & $\times$ & & & & & & & & & & & \\
\hline & Mendes et al. (2004) & $x$ & & & & & & & & & & & \\
\hline
\end{tabular}

\footnotetext{
${ }^{1}$ Residuos de planta desalinizadora, ${ }^{2}$ Residuos de producción de leche, ${ }^{3}$ Residuos del cultivo de tomate
} 


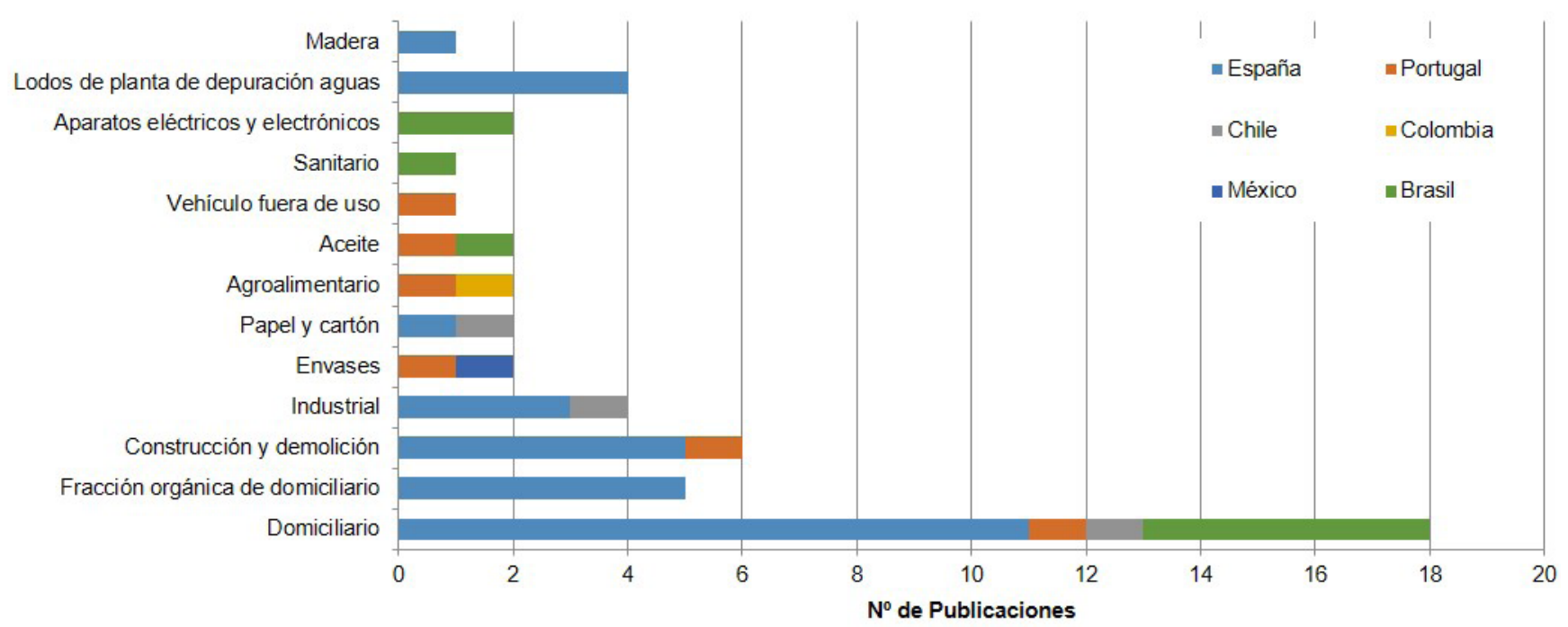

Fig. 1. Relación del número de publicaciones por país y tipo de residuo

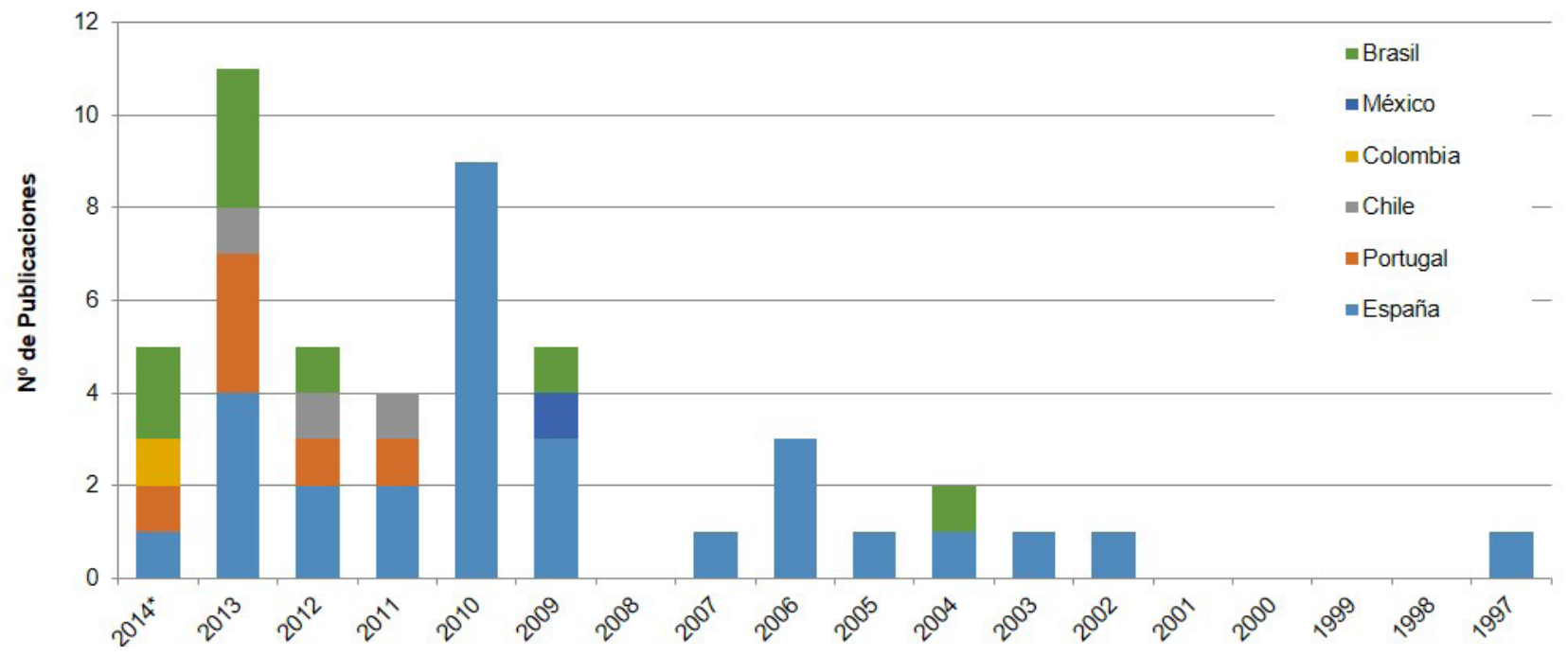

Fig. 2. Evolución temporal del número de publicaciones por país

impacto. Se ha aplicado en todos los casos el método CML (Guinée 2002) para la aplicación de los factores de ponderación de las categorías de impacto: acidificación, eutrofización, calentamiento global, reducción de la capa de ozono, disminución del recurso abiótico y oxidación fotoquímica. Finalmente, se interpretan los resultados en función de los objetivos marcados en cada caso de estudio.

\section{Gestión de residuos domiciliarios y sistemas de recogida selectiva (España).}

El objetivo principal de este caso de estudio que se presenta fue analizar el desempeño ambiental de uno de los sistemas de gestión de residuos más implantados en España. Concretamente, se analiza un sistema de gestión con recogida selectiva de cuatro fracciones: papel-cartón, vidrio y envases ligeros en área de aportación, y fracción resto a nivel de acera.

La figura 3 representa gráficamente los límites del sistema en forma de entradas y salidas de materia y energía. Además, en dicha figura también se distinguen las diferentes etapas del sistema de gestión de residuos, a considerar: pre-recogida, recogida y transporte, pre-tratamiento, tratamiento y disposición final.

Cabe señalar que quedan fuera del alcance de este estudio las infraestructuras necesarias para el desarrollo de cada etapa.

La unidad funcional considerada en este caso es la gestión de 1 tonelada de residuo domiciliario con la composición prersentada en el cuadro II en una 


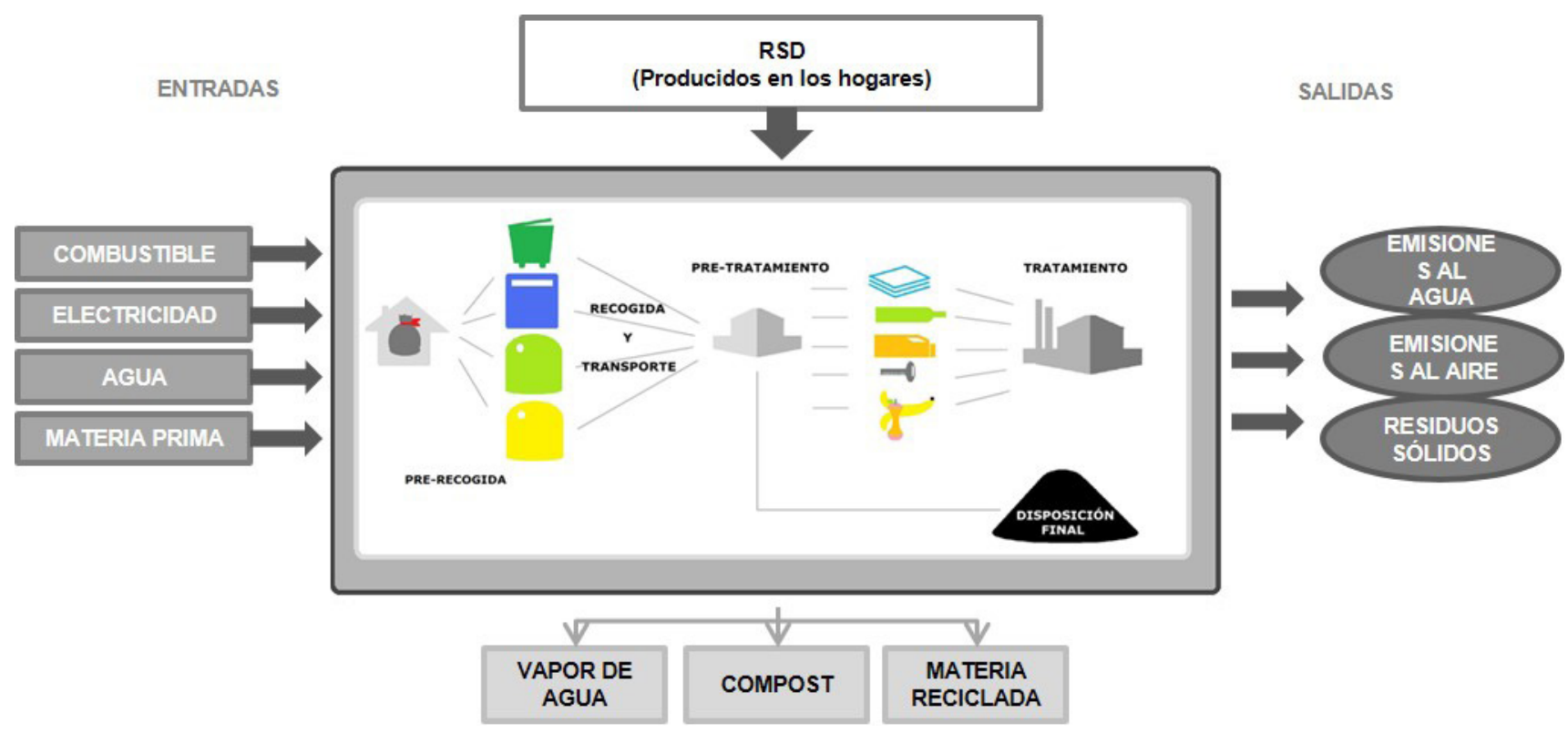

Fig. 3. Alcance del estudio del caso de aplicación en España

CUADRO II. Composición de los residuos en el caso de aplicación de España (PNIR 2008-2015).

\begin{tabular}{ccccccccc}
\hline Fracción & Materia orgánica & Papel-Cartón & Plástico & Vidrio & Férrico & No férrico & Madera & Otros \\
$\%$ & 44 & 21 & 10.6 & 7 & 3.4 & 0.7 & 1 & 12.3 \\
\hline
\end{tabular}

población española tipo de aproximadamente 200000 habitantes.

En el sistema de gestión que se evalúa se recogen fracciones valorizables de vidrio, papel-cartón y envases, que tras pasar por plantas de clasificación se destinan a su reciclaje. La fracción resto, recogida a nivel de acera, tras pasar por una estación de transferencia (ET), se envía a una planta de recuperación de materiales (PRM), donde se separa la fracción orgánica del resto de residuos para ser llevada, posteriormente, a una planta de compostaje. Finalmente, los rechazos que se van obteniendo en las diferentes instalaciones, se compactan en balas y se envían a un vertedero sin recuperación energética. Se consideran las cargas evitadas por la producción del compost y los materiales reciclados obtenidos.

Teniendo en cuenta la composición de los residuos, las tasas de recogida de cada fracción, así como la eficiencia de las instalaciones de pre-tratamiento y tratamiento de los residuos descritos en Bovea et al. (2010), se obtiene el modelo a analizar mostrado en la figura 4.

Con el fin de conseguir un inventario adaptado al caso de estudio, se obtuvieron datos primarios directamente de empresas españolas encargadas de la gestión de los residuos para las etapas de pre-recogida, recogida, pre-tratamiento y el tratamiento a través del compostaje. Los datos de inventario correspondientes a las etapas de tratamiento mediante el reciclado y vertido son datos secundarios adaptados al caso de estudio a partir de la base de datos Ecoinvent (2008).

El cuadro III detalla los datos de inventario utilizados para cada etapa del ciclo de vida de la gestión de los residuos.

Los datos minoritarios, como los procesos de producción de la energía, de obtención del diesel, de fabricación de materiales, etc. se han adaptado de Ecoinvent (2008). Por otro lado, el mix eléctrico considerado, mostrado en el cuadro IV, es el correspondiente a España en el año 2012.

La figura 5 muestra la contribución de cada etapa del ciclo de vida del sistema de gestión de residuos, a cada categoría de impacto.

En general, se observa que el reciclaje permite evitar carga contaminante en tres de las cinco categorías de impacto analizadas (acidificación, eutrofización y oxidación fotoquímica), ya que evita el consumo de materia prima virgen. Por otro lado, el combustible consumido durante la recogida y transporte de los residuos provoca que dicha etapa contribuya a aumentar el impacto, prácticamente en todas las categorías analizadas, principalmente 


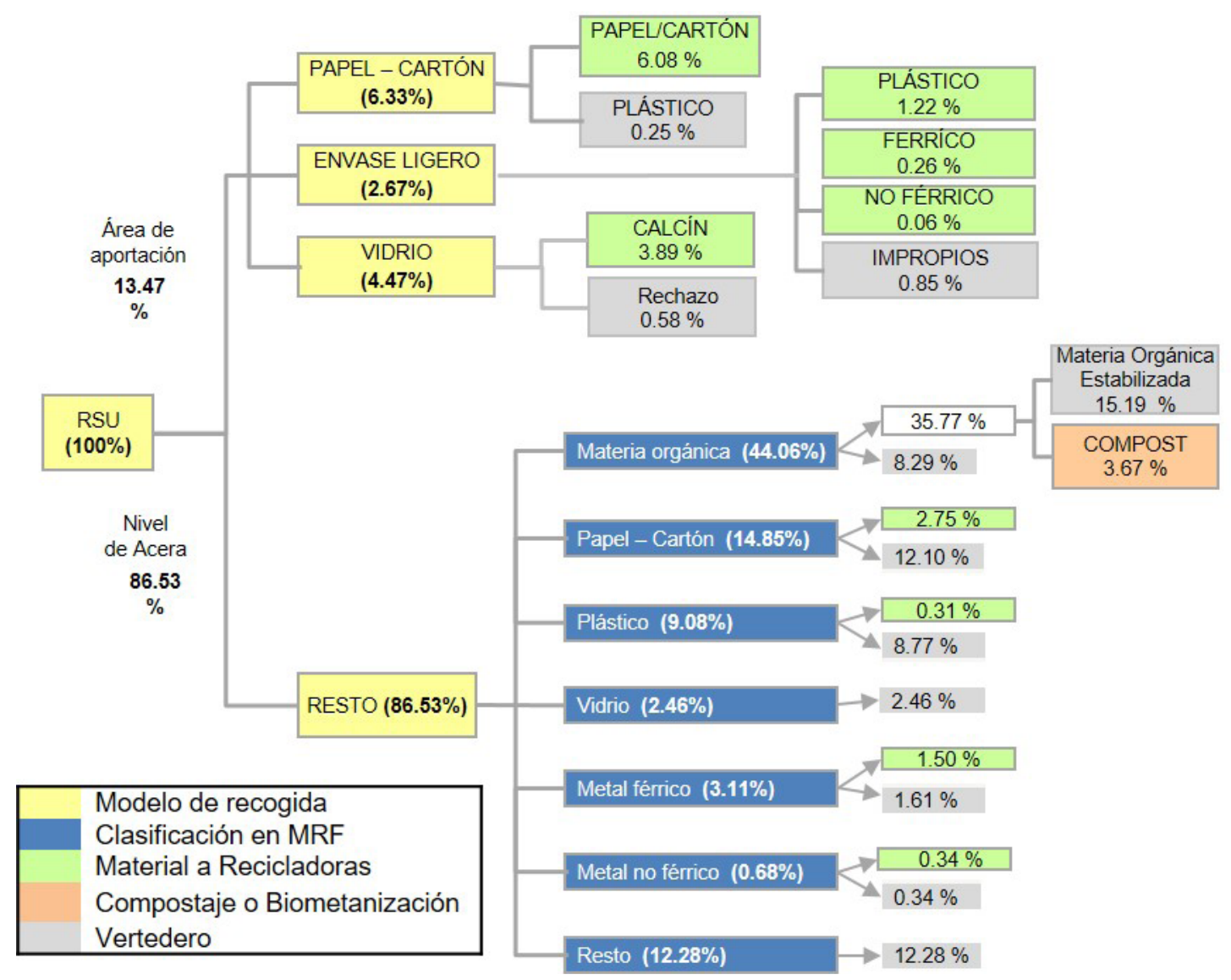

Fig. 4. Modelo del sistema de gestión de residuos domiciliario correspondiente al caso de aplicación en España

en acidificación y deterioro de la capa de ozono. La etapa de vertido contribuye significativamente al aumento de todas las categorías de impacto, siendo esta especialmente significativa para el calentamiento global, la oxidación fotoquímica y la eutrofización. Para reducir dicho impacto, medidas como la recuperación energética, a partir de la combustión de los gases producidos en el vertedero, permitirían mejorar significativamente el comportamiento global del sistema.

Gestión de residuos de aparatos eléctricos y electrónicos correspondientes a terminales de telefonía móvil (México).

El objetivo principal de este caso de estudio fue analizar el impacto ambiental del ciclo de vida de un terminal tipo de telefonía móvil, prestando especial atención a la etapa de gestión de residuos. Para ello, se propusieron dos escenarios de fin de vida, uno considerando que no se realiza ninguna gestión y que el terminal se deposita en vertedero junto con el resto de residuos domiciliarios de los hogares, y otro considerando su correcta gestión como residuo de aparato eléctrico y electrónico (RAEE) y atendiendo a las características de esta gestión en México.

Las etapas que configuran el ciclo de vida del sistema a analizar fueron:

- Adquisición y la producción de materias primas. Con el fin de caracterizar el residuo procedente de los terminales de telefonía móvil y obtener así su composición promedio, se realizó una campaña de recogida de teléfonos móviles en desuso, que fueron desensamblados y caracterizados con el fin de obtener su composición en cuanto a materiales. En la fase de desensamblado se separaron las partes para identificar el material, proceso de fabricación, peso, etc. de cada componente. Este proceso se completó con datos bibliográficos para establecer la composición media de componentes de pequeño tamaño tales como altavoces, cámara fotográfica, micrófono y antena. La composición final obtenida para un terminal de telefonía móvil promedio es la detallada en el cuadro $\mathbf{V}$. No se consideró el embalaje del producto terminado. 
CUADRO III. DATOS DE INVENTARIO CORRESPONDIENTES AL CASO DE APLICACIÓN DE ESPAÑA

\begin{tabular}{|c|c|c|c|c|c|c|}
\hline \multirow[t]{2}{*}{ Etapa } & \multirow[t]{2}{*}{ Impacto } & \multirow[t]{2}{*}{ Carga evitada } & \multicolumn{4}{|c|}{ DATOS DE INVENTARIO } \\
\hline & & & FRACCIÓN & Bolsas/t & Contenedor/t & \\
\hline \multirow{5}{*}{ Pre-recogida } & \multirow{5}{*}{$\begin{array}{l}\text { Utilización de bolsas de } \\
\text { PEBD extrusionadas y } \\
\text { contenedores de PEAD y } \\
\text { acero galvanizado }^{(1)}\end{array}$} & \multirow{5}{*}{ Nula } & Resto & 501.25 & $4.24 \mathrm{E}-3$ & \\
\hline & & & Orgánica & 501.25 & $4.24 \mathrm{E}-3$ & \\
\hline & & & Vidrio & 168.42 & $5.22 \mathrm{E}-4$ & \\
\hline & & & Papel-Cartón & 657.89 & $2.04 \mathrm{E}-3$ & \\
\hline & & & Envases & 1503.76 & $4.66 \mathrm{E}-3$ & \\
\hline \multirow{2}{*}{$\begin{array}{l}\text { Recogida y } \\
\text { transporte }^{(2)}\end{array}$} & \multirow{2}{*}{$\begin{array}{l}\text { Consumo de } \\
\text { combustible }\end{array}$} & \multirow[b]{2}{*}{ Nula } & Camión de media carga & \multicolumn{3}{|c|}{$0.02561 / \mathrm{t} \cdot \mathrm{km}$} \\
\hline & & & Camión de gran carga & & $0.0146 \mathrm{l} / \mathrm{t} \bullet \mathrm{km}$ & \\
\hline \multirow{6}{*}{ Pre-tratamiento } & \multirow{3}{*}{$\begin{array}{l}\text { Consumo de combustible } \\
\text { y electricidad: }\end{array}$} & \multirow{6}{*}{ Nula } & PLANTA & $\begin{array}{l}\text { Electricidad } \\
(\mathrm{kWh} / \mathrm{t})\end{array}$ & Gasóleo (1/t) & $\begin{array}{l}\text { Agua } \\
(\mathrm{m} 3 / \mathrm{t})\end{array}$ \\
\hline & & & Clasificadora de vidrio & 8.05 & 0.53 & - \\
\hline & & & Clasificadora de papel-cartón & 3.99 & 2.58 & - \\
\hline & - Clasificación de las & & Clasificadora de envases & 6.10 & 0 & $\mathrm{n} / \mathrm{d}$ \\
\hline & $\begin{array}{l}\text { fracciones de vidrio, } \\
\text { papel-cartón y envases } \\
\text { recogidos de forma } \\
\text { selectiva. }\end{array}$ & & Estación de transferencia & 1.36 & 1.76 & 0.043 \\
\hline & $\begin{array}{l}\text { - Transferencia y clasi- } \\
\text { ficación de la fracción } \\
\text { resto recogida en área de } \\
\text { aportación. }\end{array}$ & & $\begin{array}{l}\text { Planta de recuperación de } \\
\text { material (PRM) }\end{array}$ & 8.11 & 0.56 & 0.004 \\
\hline \multirow{3}{*}{$\begin{array}{l}\text { Tratamiento } \\
\text { (reciclaje y } \\
\text { compostaje) }\end{array}$} & $\begin{array}{l}\text { Consumos de combusti- } \\
\text { ble y electricidad en las } \\
\text { operaciones de reciclaje }\end{array}$ & $\begin{array}{l}\text { Material } \\
\text { virgen corres- } \\
\text { pondiente a } \\
\text { cada fracción } \\
\text { reciclada }\end{array}$ & \multicolumn{4}{|c|}{ Datos secundarios adaptados de Ecoinvent (2008) } \\
\hline & & & PLANTA & $\begin{array}{l}\text { Electricidad } \\
(\mathrm{kWh} / \mathrm{t})\end{array}$ & Gasóleo (1/t) & $\begin{array}{l}\text { Agua } \\
(\mathrm{m} 3 / \mathrm{t})\end{array}$ \\
\hline & $\begin{array}{l}\text { Consumos de } \\
\text { combustible y } \\
\text { electricidad en las } \\
\text { operaciones de } \\
\text { compostaje }\end{array}$ & $\begin{array}{l}\text { Fertilizante } \\
\text { químico }\end{array}$ & Planta de compostaje & 19.67 & $0.30 \mathrm{w}$ & 0.05 \\
\hline $\begin{array}{l}\text { Disposición } \\
\text { final }\end{array}$ & $\begin{array}{l}\text { Consumos de combusti- } \\
\text { ble en las operaciones de } \\
\text { movimiento del residuo } \\
\text { en vertedero }\end{array}$ & Nula & Datos secundarios adaptados d & Ecoinvent $(2$ & 08) & \\
\hline
\end{tabular}

(1) El proceso de fabricación de bolsas y contenedores pueden consultarse en Bovea et al. (2010)

${ }^{(2)}$ Las distancias consideradas entre instalaciones pueden consultarse en Bovea et al. (2010)

$\mathrm{n} / \mathrm{d}$ : dato no disponible 
CUADRO IV. MIX ELÉCTRICO CORRESPONDIENTE AL CASO DE APLICACIÓN DE ESPAÑA, PARA EL AÑO 2012

\begin{tabular}{lccc}
\hline FUENTE & Porcentaje (\%) & FUENTE & $\begin{array}{c}\text { Porcentaje } \\
(\%)\end{array}$ \\
\hline Nuclear & 22 & Hidráulica & 16 \\
Carbón & 7 & Eóliwca & 16 \\
Fuel & 1 & Fotovoltaica & 4 \\
Ciclo combinado & 23 & Gas natural & 11 \\
\hline
\end{tabular}

Fuente. Red Eléctrica Española (REE) 2013

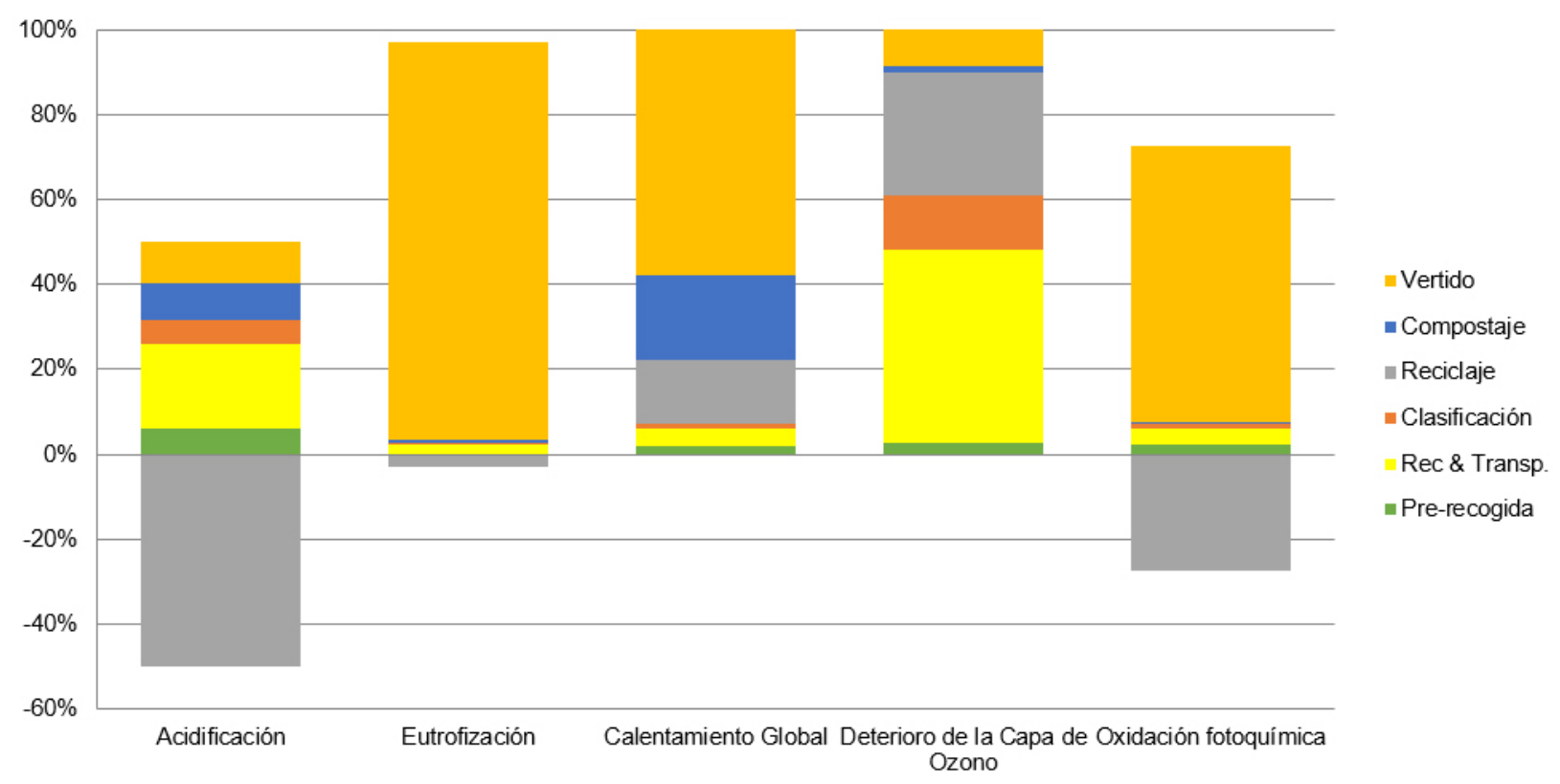

Fig. 5. Evaluación del impacto correspondiente al caso de aplicación de España

- Distribución. Asumiendo la producción del móvil en China y su trasporte a México. Considerando su transporte en barco transoceánico (Shangai-Ensenada, México: $10771 \mathrm{~km}$ ) y transporte terrestre (Ensenada-Cd. México-Mexicali: 5589km)

- Uso. De acuerdo con Rosen y Meier's (2000), el consumo de un teléfono móvil es de aproximadamente $9 \mathrm{kWh} /$ año. Se consideró la aportación energética por tecnología (mix eléctrico), correspondiente a México en el año 2013-2014, según indica el cuadro VI.

- Fin de vida. Se consideraron dos escenarios:

o S1: Incluye el depósito del terminal de telefonía móvil en vertedero, sin previo tratamiento.

o S2: Incluye el proceso de descontaminación y separación manual además de tratamientos distintos para la recuperación de materiales de las distintas fracciones. En esta etapa se asume una tasa de reciclaje de: plástico $21.53 \%$, férricos $12.86 \%$ y no férricos $9.77 \%$.
La unidad funcional considerada para este caso es el ciclo de vida de un terminal de telefonía móvil promedio, con un uso de 30 meses. Cabe señalar que quedan fuera del alcance de este estudio las infraestructuras necesarias para el desarrollo de cada etapa.

Para configurar el inventario adaptado a este caso de estudio (Cuadro VII) se tomó información de la base de datos Ecoinvent (2008) para todas las etapas, excepto vertedero, que se tomó el modelo propuesto por McDougall et al. (2001) de acuerdo con la base de datos BUWAL250, adaptado a cada una de las fracciones que componen el RAEE. En ambos casos, se ha realizado una adaptación previa de los datos al caso de estudio situado en México (aportación energética por tecnologías, distancias, medios de transporte, etc.).

En la etapa de fin de vida, se recuperan los materiales mostrados en la figura 6. Para el escenario $\mathrm{S} 1$, que considera el vertido, no se recupera ninguna fracción. Para el escenario S2, que incluye el 
CUADRO V. COMPOSICIÓN PROMEDIO DE UN TERMINAL DE TELEFONÍA MÓVIL

\begin{tabular}{lccccc}
\hline Fracción & Plástico & Férrico & No Férrico & Batería & PCI-PCL* \\
\hline$\%$ & 39.96 & 2.41 & 5.04 & 25.69 & 26.90 \\
\hline
\end{tabular}

*PCI: Placa de Circuito Impreso; PCL: Pantalla de Cristal Líquido

CUADRO VI. MIX ELÉCTRICO CONSIDERADO EN EL CASO DE APLICACIÓN DE MÉXICO, PARA EL AÑO $2013-2014$

\begin{tabular}{lclc}
\hline Mix & Porcentaje (\%) & Mix & Porcentaje $(\%)$ \\
\hline Termoeléctrica & 19.16 & Nucleoeléctrica & 4.74 \\
Ciclo combinado & 50.4 & Eólica & 0.75 \\
Dual & 6.21 & Hidroeléctrica & 12.34 \\
Carboeléctrica & 6.40 & Fotovoltaica & 0.005 \\
\hline
\end{tabular}

Fuente: SENER 2014

CUADRO VII. DATOS DE INVENTARIO CORRESPONDIENTES AL CASO DE APLICACIÓN DE MÉXICO

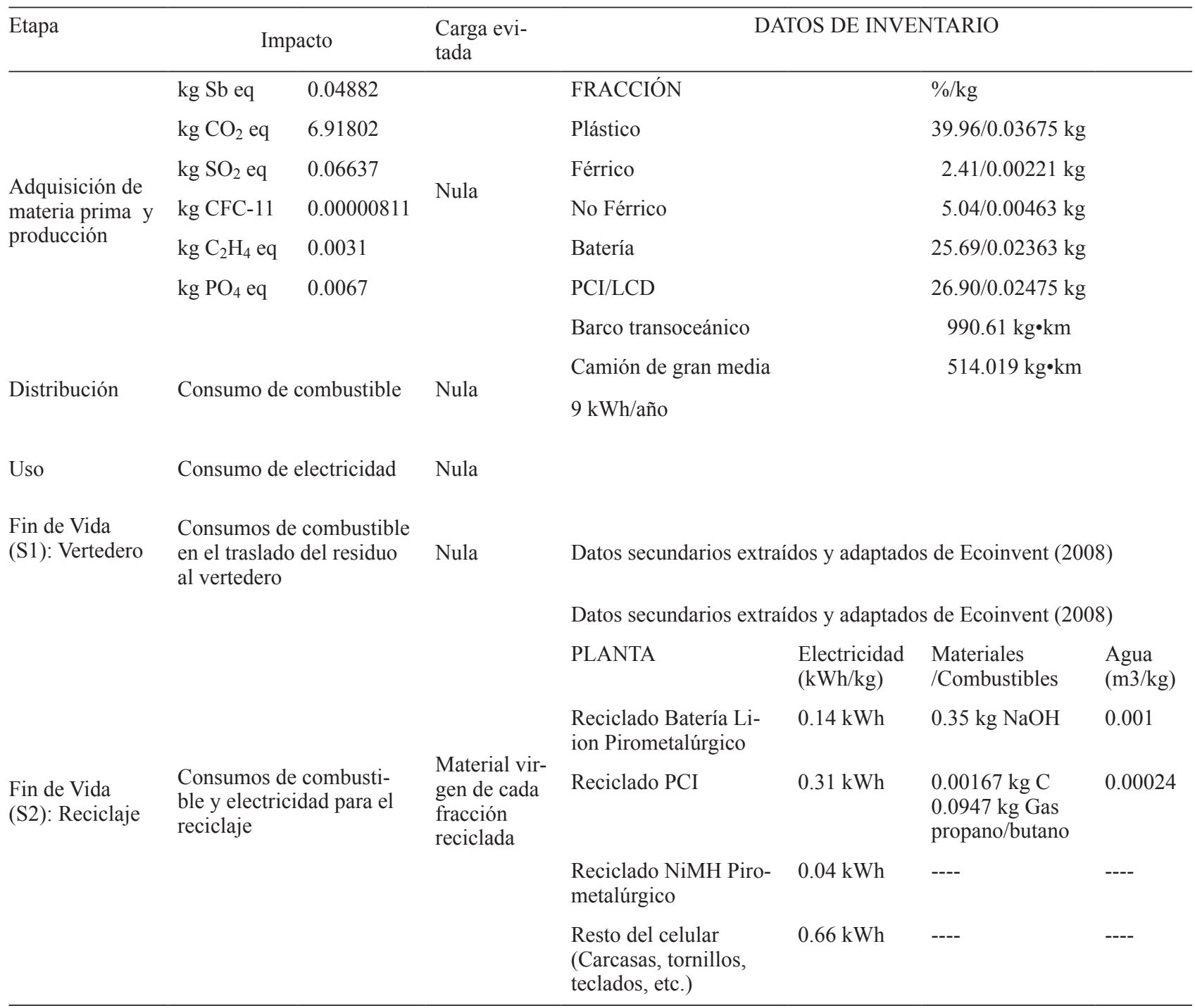




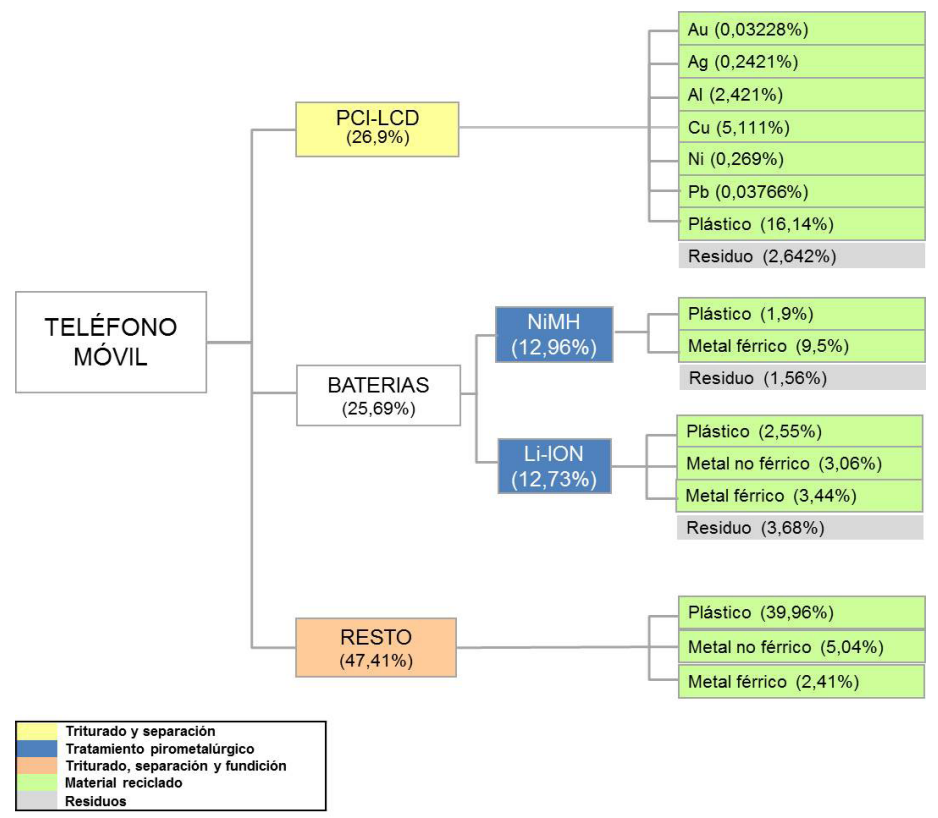

Fig. 6. Datos del modelo de inventario considerado para el caso de aplicación de México

Escenario S1

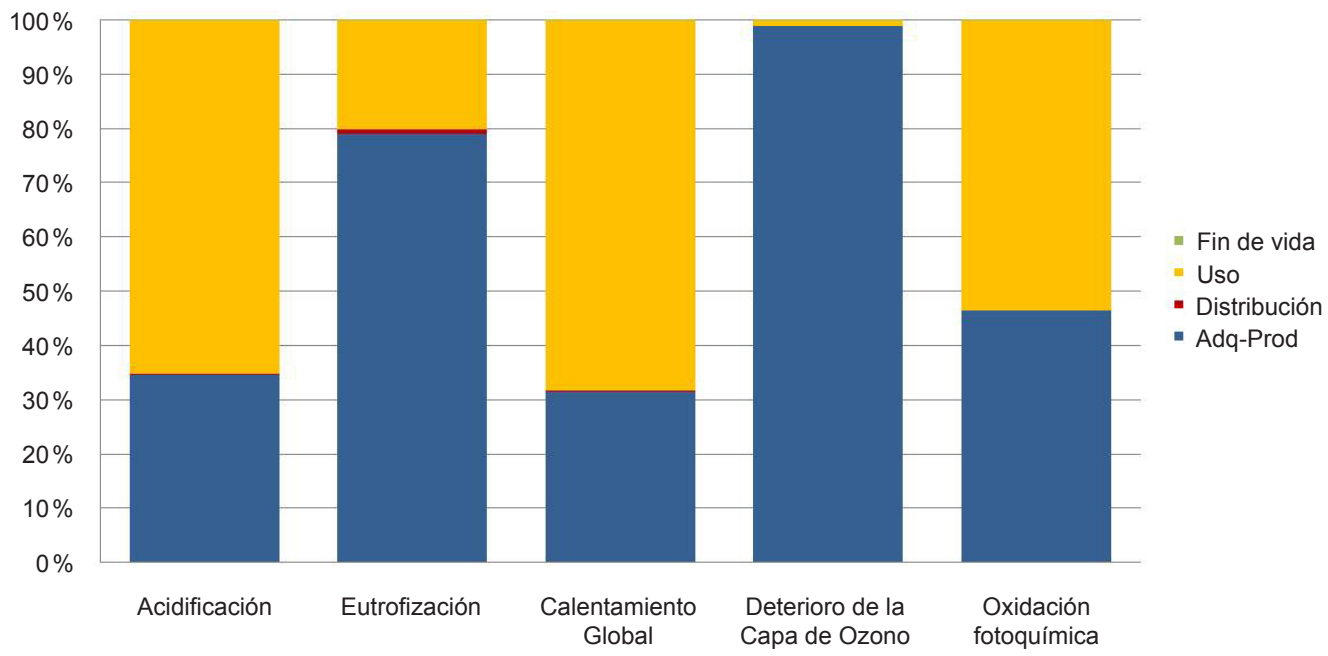

Fig. 7. Evaluación del impacto correspondiente al caso de aplicación de México: Escenario S1

reciclado del terminal, se considera que, tras pasar por un proceso de trituración y separación mecánica y manual (Ecoinvent 2008) se recuperan los materiales indicados, asumiendo una tasa de eficiencia en recuperación de material reciclable 1:1.

Las figuras 7 y 8 muestran los resultados de los indicadores ambientales relativos obtenidos para cada categoría de impacto y distribuidos por las diferentes etapas del ciclo de vida consideradas, para cada escenario analizado.

Se observa que la etapa de adquisición de materiales/producción y uso, son las etapas del ciclo de vida que mayor contribución tienen para todas las categorías de impacto en ambos escenarios. El impacto de la etapa de fin de vida no aparece como significativo para ninguna de las categorías de impacto, según los modelos de inventario actualmente incorporados en las bases de datos comerciales. Sin embargo, sí se observa que la incorporación del reciclaje de las fracciones recuperadas permite compensar parte del impacto del resto de etapas.

Analizando más en detalle la etapa de fin de vida, la figura 9 muestra la comparativa entre ambos escenarios. 


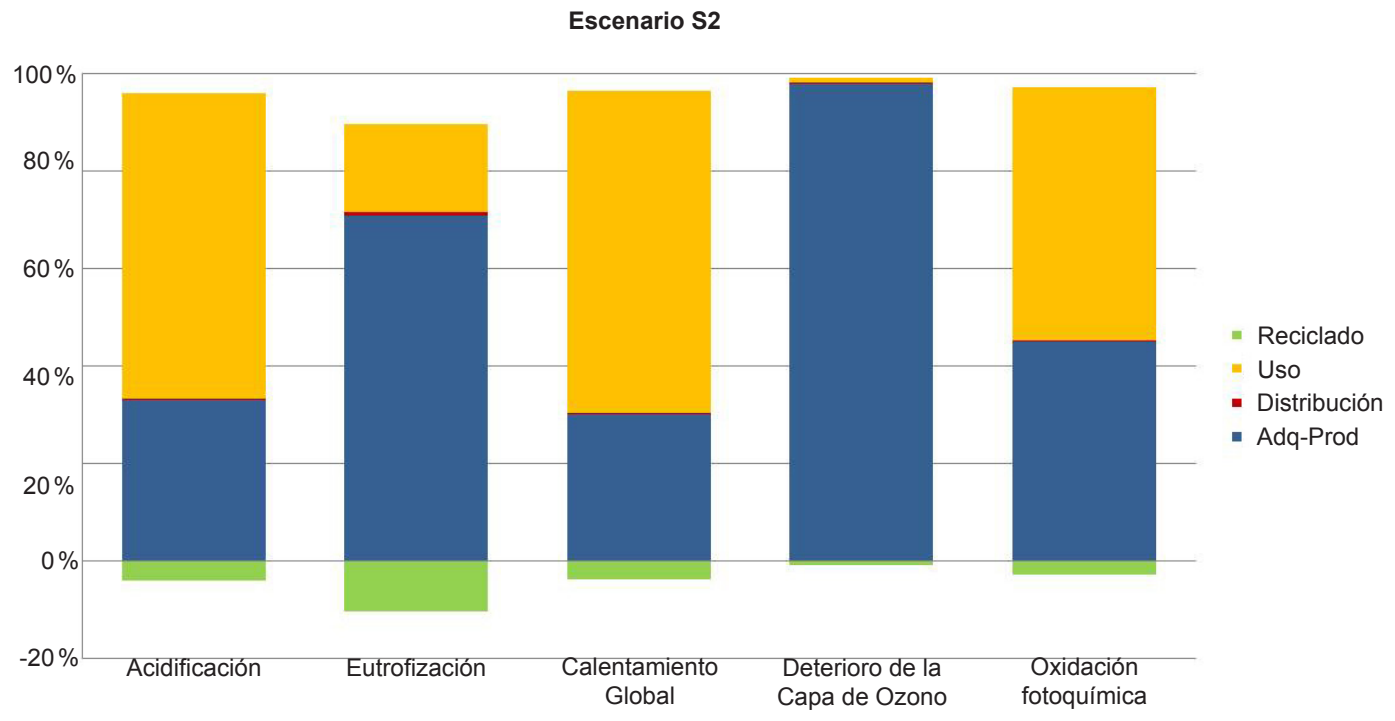

Fig. 8. Evaluación del impacto: Escenario S2

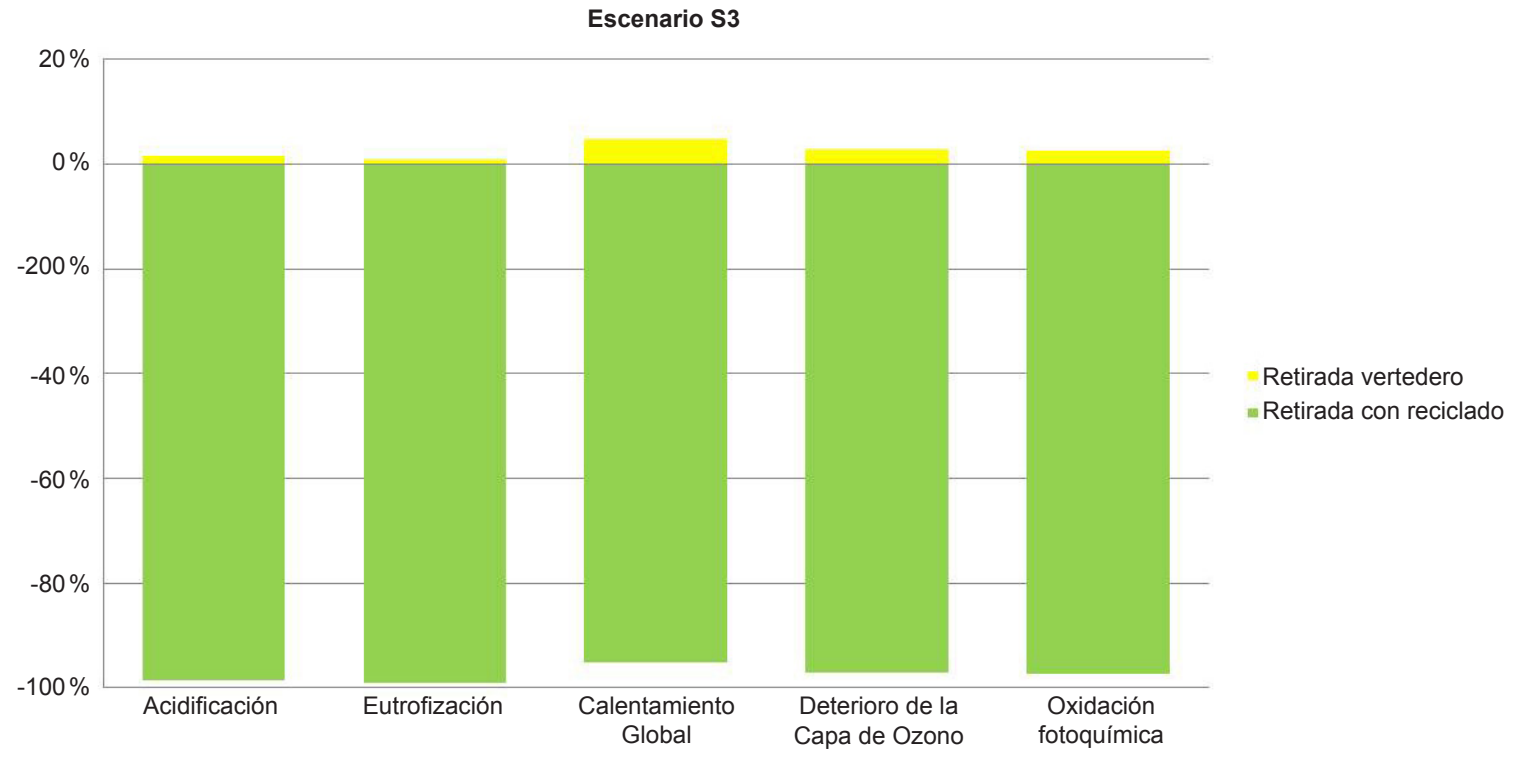

Fig. 9. Comparación de la Etapa de Fin de Vida del teléfono móvil correspondiente al caso de aplicación de México (Vertido vs Reciclaje)

En general, se observa que la etapa de adquisición de materiales, seguida de la de uso, son las etapas que presentan una mayor contribución al impacto a lo largo del ciclo de vida de un terminal de telefonía móvil, siendo los componentes eléctricos y electrónicos del teléfono móvil (batería y la placa de circuito impreso) los causantes de ello.

En la etapa de gestión de los residuos electrónicos, el reciclaje juega un papel clave, no sólo porque reduce el impacto ambiental debido a los tratamientos, sino también porque ahorra recursos limitados y la energía necesaria para su extracción a través de la recuperación de los materiales que se encuentran en ellos. Sin embargo, en este tipo de productos, es necesario analizar otras estrategias de fin de vida previas al reciclaje que actualmente se promueve, como su preparación para su reutilización o la extensión de la vida útil.

Desde el punto de vista de la aplicación de la metodología ACV, sería conveniente invertir esfuerzos en la obtención de modelos de inventario, tanto para el vertido como para el reciclaje, adaptados al caso de los residuos procedentes de componentes eléctricos y electrónicos, ya que los actuales no consideran 


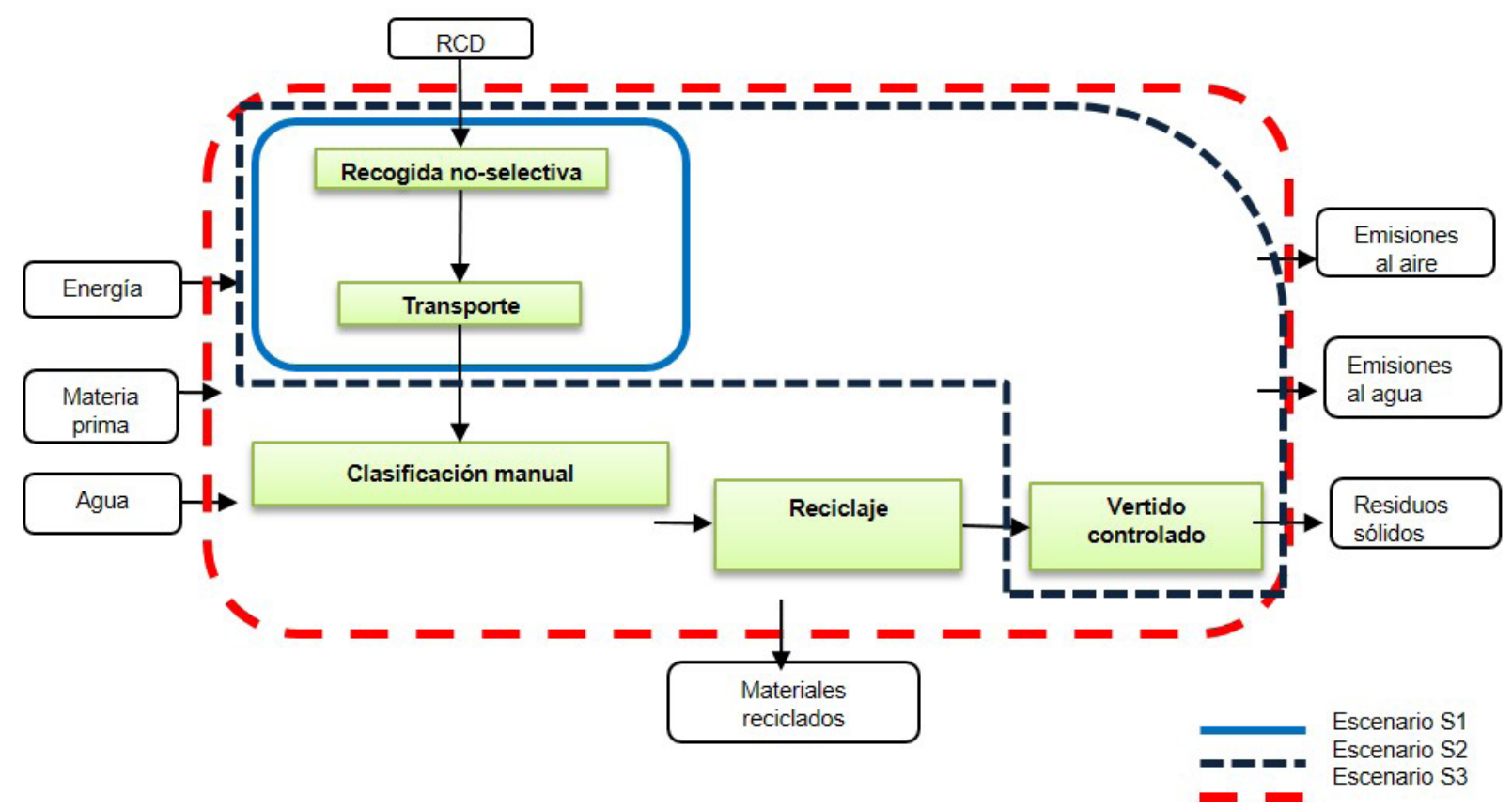

Fig. 10. Alcance del estudio del caso de aplicación de Argentina

CUADRO VIII. COMPOSICIÓN DE LOS RCD CONSIDERADOS EN EL CASO DE APLICACIÓN DE ARGENTINA

\begin{tabular}{llllll}
\hline Fracción & Inerte & Papel-Cartón & Metales & Madera & Otros \\
\hline$\%$ & 75 & 0.3 & 2.5 & 4 & 18.2
\end{tabular}

Fuente MARM 2008

estas fracciones en toda su extensión. Es importante resaltar también, la necesidad de incorporar las cargas ambientales evitadas, tal y como se ha realizado en este caso de estudio.

\section{Gestión de residuos de construcción y demolición (Argentina)}

El objetivo de este caso de estudio fue evaluar el desempeño ambiental de posibles alternativas a la actual gestión de los residuos de construcción y demolición (RCD) en la localidad de Las Heras, provincia de Mendoza (Argentina). Para ello, se elaboró y analizó un inventario del ciclo de vida de la gestión de los RCD aplicable a la zona de estudio, considerando datos primarios predominantemente. Posteriormente, se analizó el perfil ambiental de alternativas de gestión con incorporación de reciclaje y disposición final controlada.

Para este caso se consideraron los tres escenarios de gestión mostrados en la figura 10:

- Escenario S1: corresponde a la situación actual de vertido incontrolado. Las etapas incluidas en este escenario son las de recogida y transporte, no se consideraron consumos en la etapa de vertido.

- Escenario S2: incorpora vertido controlado al escenario S1. El sitio se ubica en el vertedero de residuos sólidos urbanos localizado en el Distrito El Borbollón. Por lo tanto existen consumos debidos a la gestión del residuo en vertedero.

- Escenario S3: considera la instalación de una planta de clasificación y reproceso de inertes (PCRI) en el mismo lugar donde se encuentra el vertedero actual. Este escenario incluye el reciclaje de las fracciones inertes, metales, papel-cartón y madera, y son consideradas las cargas evitadas correspondientes a cada fracción.

La unidad funcional fue la gestión de 1 tonelada de RCD, adoptando la composición indicada en el cuadro VIII, dado que no existen aún datos a nivel regional. No obstante, por las características de las construcciones en Mendoza con predominancia de materiales inertes, y con base a datos propios de composición (Mercante 2005), se puede asumir que la composición adoptada es muy cercana a la real.

Se elaboró un modelo de inventario propio a partir de datos recopilados en empresas de la cadena de 
CUADRO IX. DATOS DE INVENTARIO CORRESPONDIENTES AL CASO DE APLICACIÓN DE ARGENTINA

\begin{tabular}{|c|c|c|c|c|c|}
\hline Etapa & Impacto & Carga evitada & \multicolumn{3}{|c|}{ DATOS DE INVENTARIO } \\
\hline \multirow{3}{*}{ Pre-recogida } & \multirow{3}{*}{$\begin{array}{l}\text { Utilización de contenedores } \\
\text { metálicos }\end{array}$} & \multirow{3}{*}{ Nula } & FRACCIÓN & Contenedor de $3 \mathrm{~m}^{3} / \mathrm{t}$ & Contenedor $5 \mathrm{~m}^{3} / \mathrm{t}$ \\
\hline & & & & & \\
\hline & & & Mixta & $6.667 \mathrm{E}-05$ & $4.000 \mathrm{E}-05$ \\
\hline \multirow{2}{*}{$\begin{array}{l}\text { Recogida y } \\
\text { transporte }\end{array}$} & \multirow{2}{*}{ Consumo de combustible } & \multirow{2}{*}{ Nula } & \multicolumn{2}{|c|}{ Transporte contenedor de $3 \mathrm{~m} 3$} & $0.0756 \mathrm{l} / \mathrm{t} \cdot \mathrm{km}$ \\
\hline & & & \multicolumn{2}{|c|}{ Transporte contenedor de $5 \mathrm{~m} 3$} & $0.03351 / \mathrm{t} \cdot \mathrm{km}$ \\
\hline & Consumo de combustible & & PLANTA & Electricidad $(\mathrm{kWh} / \mathrm{t})$ & Gasóleo (1/t) \\
\hline Pre-tratamiento & $\begin{array}{l}\text { y electricidad en la clasifi- } \\
\text { cación de las fracciones de } \\
\text { madera, metales, papel-cartón } \\
\text { e inertes }\end{array}$ & Nula & Clasificación & -- & 0.05 \\
\hline \multirow{6}{*}{$\begin{array}{l}\text { Tratamiento } \\
\text { (reciclaje) }\end{array}$} & \multirow{4}{*}{$\begin{array}{l}\text { Consumos de combustible } \\
\text { y electricidad en las cargas } \\
\text { evitadas }\end{array}$} & \multirow{6}{*}{$\begin{array}{l}\text { Material virgen } \\
\text { correspondiente } \\
\text { a cada fracción } \\
\text { reciclada }\end{array}$} & FRACCIÓN & $\begin{array}{l}\text { Electricidad } \\
\text { (kWh/ton) }\end{array}$ & Gasóleo (1/t) \\
\hline & & & Inerte & 0.34 & 0.70 \\
\hline & & & Resto de fracciones & $\begin{array}{l}\text { Datos secundarios extr } \\
\text { de Ecoinvent (2008) }\end{array}$ & aídos y adaptados \\
\hline & & & FRACCIÓN & Electricidad (kWh/t) & Gasóleo (1/t) \\
\hline & \multirow{2}{*}{$\begin{array}{l}\text { Consumos de combustible y } \\
\text { electricidad en las operacio- } \\
\text { nes de reproceso }\end{array}$} & & Inertes & 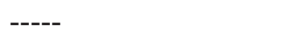 & 0.60 \\
\hline & & & Resto de fracciones & \multicolumn{2}{|c|}{$\begin{array}{l}\text { Datos secundarios extraídos y adaptados } \\
\text { de Ecoinvent (2008) }\end{array}$} \\
\hline \multirow{2}{*}{$\begin{array}{l}\text { Disposición } \\
\text { final }\end{array}$} & \multirow{2}{*}{$\begin{array}{l}\text { Consumos de combustible en } \\
\text { las operaciones de gestión del } \\
\text { vertedero }\end{array}$} & \multirow[b]{2}{*}{ Nula } & Gasóleo (1/t) & & \\
\hline & & & 0.15 & & \\
\hline
\end{tabular}

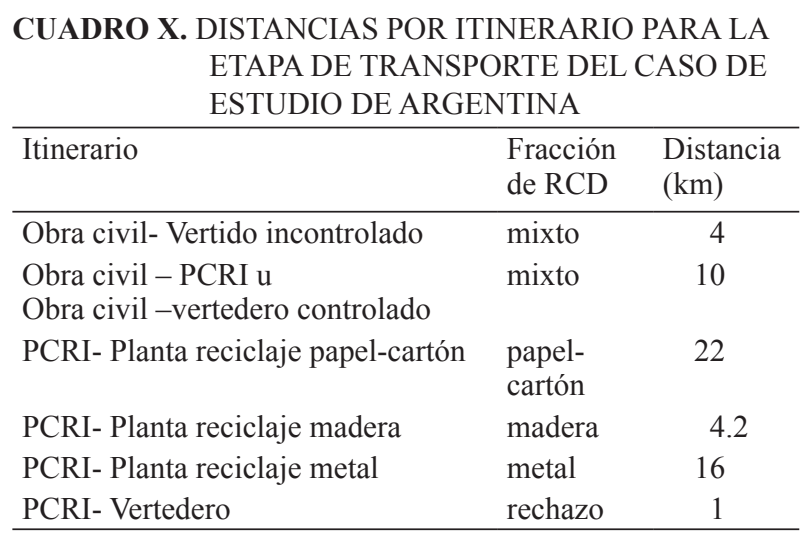

gestión de RCD siempre que fue posible, tal y como muestra el cuadro IX, y completando con datos de Ecoinvent (2008), previa su adaptación al caso de estudio. En relación a los datos de las plantas de clasificación, los datos de inventario se han obtenido de un estudio de campo en diferentes plantas instaladas en Latinoamérica, con el fin de dar un enfoque regional-local a las tecnologías propuestas.

En la etapa de transporte, las distancias son variables dependiendo de la localización de las plantas y/o sitios de tratamiento. El cuadro $\mathbf{X}$ detalla las distancias para cada itinerario y fracción. La fracción metales se asume que se entrega a una planta de transferencia (sitio de compra-venta), la fracción de madera a una planta de fabricación de aglomerados, y la fracción papel-cartón a una planta de reciclaje.

Los datos correspondientes a los procesos de producción de la energía, de obtención del diésel y de fabricación de materiales, entre otros, se han obtenido de Ecoinvent (2008). El mix eléctrico considerado se muestra en el cuadro XI.

\section{CUADRO XI. MIX ELÉCTRICO CONSIDERADO EN EL CASO DE ESTUDIO DE ARGENTINA, PARA EL AÑO 2013}

\begin{tabular}{lcc}
\hline Tipo de Generación & Porcentaje (\%) \\
\hline Hidráulica & & 33 \\
Nuclear & & 5 \\
& Gas Natural & 44.5 \\
Térmica & Gasóleo & 8 \\
(Total 62 \%) & Carbón & 2 \\
& Fuel Oil & 7.5 \\
\hline
\end{tabular}

Fuente. CAMMESA 2012 
Escenario 1

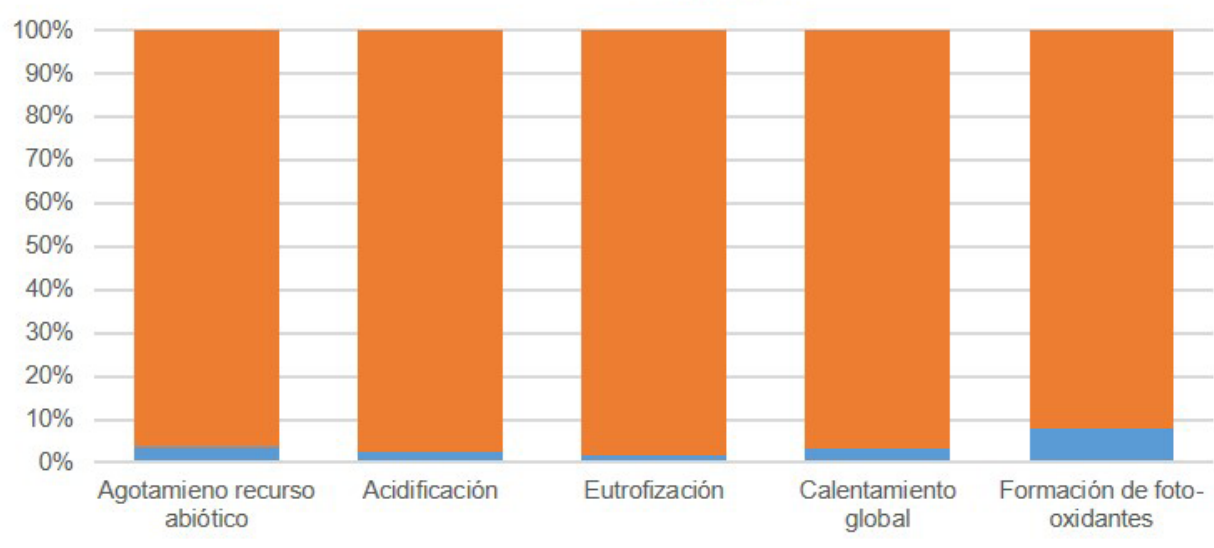

- Transporte

- Recogida

Escenario 2

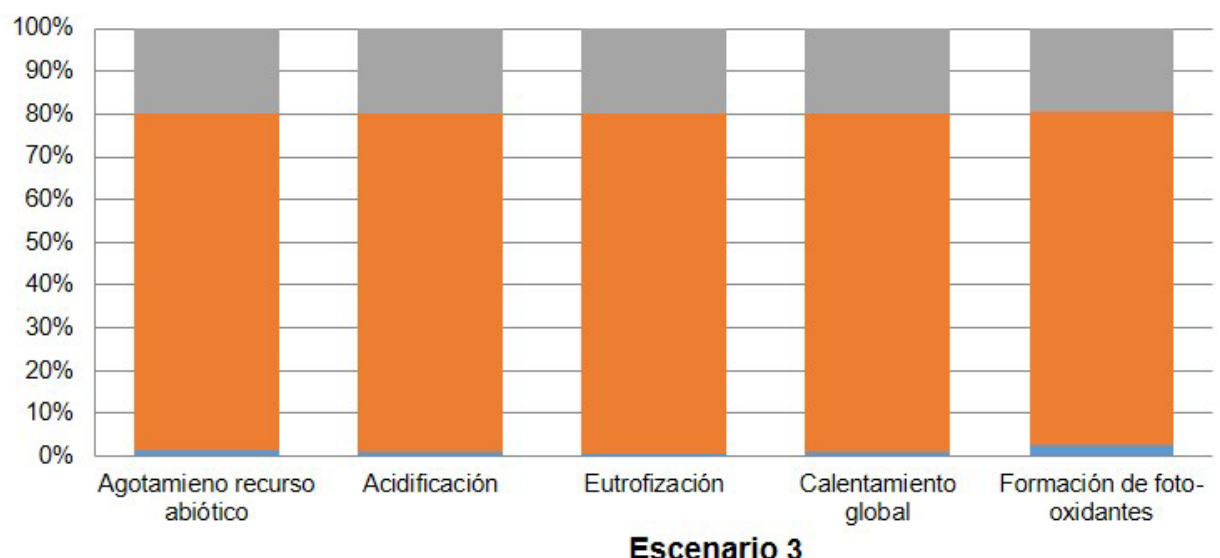

- Vertido

- Transporte

- Recogida

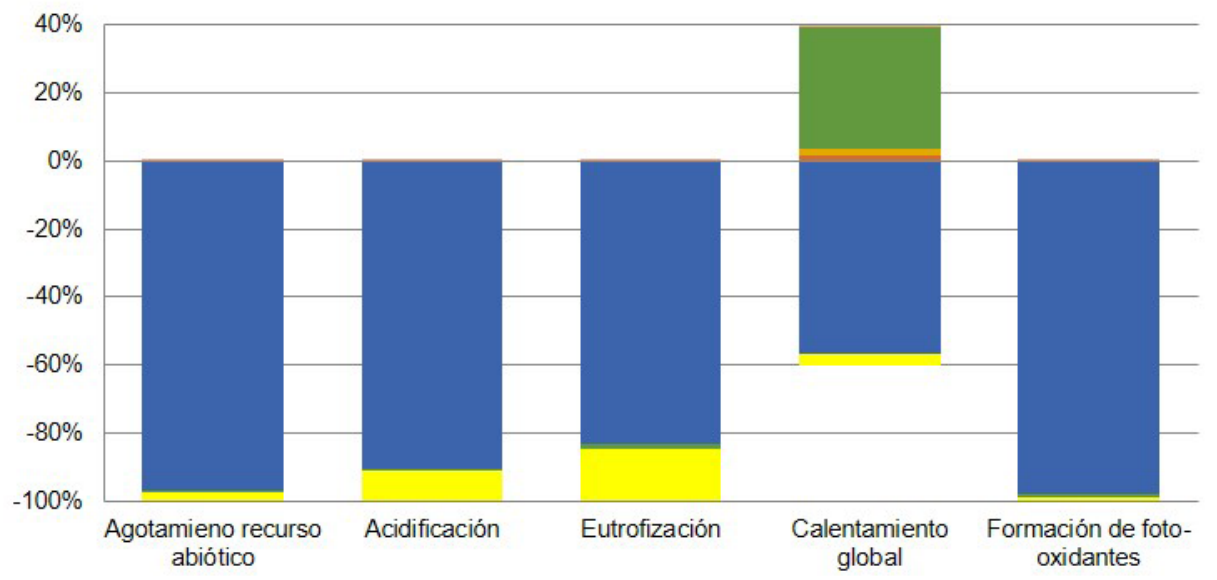

= Vertido

Reciclaje IN

- ReciclajeMD

- Reciclaje MT

- Reciclaje CP

- Clasificación

- Transporte

- Recogida

Fig. 11. Evaluación del impacto correspondiente al caso de aplicación de Argentina 


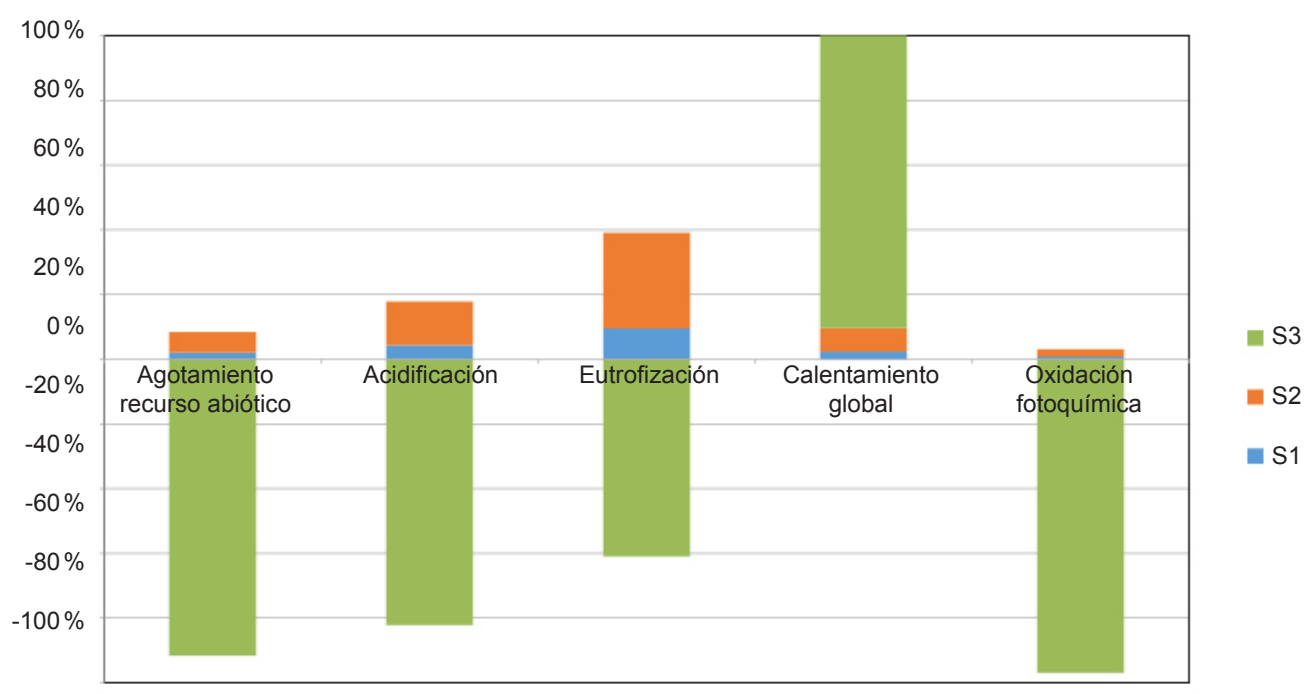

Fig. 12. Comparación del impacto ambiental de los escenarios analizados en el caso de estudio de Argentina

CUADRO XII. COMPOSICIÓN DE LOS RESIDUOS CONSIDERADOS EN EL CASO DE APLICACIÓN DE BRASIL

\begin{tabular}{lcccccc}
\hline Fracción & Materia orgánica & Papel-Cartón & Plástico & Vidrio & Metales & Otros \\
\hline$\%$ & 50.66 & 9.69 & 14.44 & 1.42 & 1.37 & 22.52 \\
\hline
\end{tabular}

La figura 11 muestra los resultados de los indicadores ambientales relativos, obtenidos para cada categoría de impacto y distribuidos en las etapas del ciclo de vida consideradas para los tres escenarios analizados.

Los resultados para los tres escenarios planteados se muestran conjuntamente en la figura 12, donde se observa que los escenarios S1 y S2 presentan perfil ambiental similar, con contribuciones netas para todas las categorías de impacto. Por otra parte, en el escenario S3, las cargas evitadas debidas al reciclaje de las diferentes fracciones valorizadas, permiten ahorros para todas las categorías de impacto excepto para la de calentamiento global, debido a la contribución neta de las fracciones de madera y papel-cartón.

\section{Gestión de residuos domiciliarios (Brasil)}

El objetivo de este caso de estudio fue analizar el desempeño ambiental de la gestión de los residuos en un municipio de Brasil, João Pessoa, que posee actualmente 723515 habitantes (IBGE, 2012) ocupando una área de $210.8 \mathrm{~km}^{2}$ y con una generación de residuos de 238050.91 t/año, que equivale a $0.90 \mathrm{~kg} / \mathrm{hab}^{*}$ día.

Hasta el año de 2003, los residuos producidos en la ciudad de João Pessoa eran depositados en un vertedero a cielo abierto sin tratamiento previo, llamado Lixão do Roger, donde cerca de 500 personas (catadores) trabajaban en condiciones precarias para separar los materiales reciclables (Nóbrega 2003). A partir de agosto de 2003 dicho vertedero fue clausurado y las personas que en él trabajaban se asociaron en una cooperativa y pasaron a trabajar en los programas de recogida selectiva implantados por la empresa de limpieza urbana. A partir de ese año, se inició una recogida selectiva de los materiales reciclables puerta a puerta, que actualmente sigue siendo todavía incipiente.

El alcance de este estudio incluye las etapas de pre-rrecogida, recogida, transporte, pre-tratamiento (separación en fracciones) y tratamiento/vertido. Se considera fuera del alcance del estudio la infraestructura necesaria en cada proceso, así como el tratamiento y posterior reciclaje del caucho y de los residuos peligrosos separados en la planta de separación de materiales.

La unidad funcional considerada en este estudio corresponde a la gestión de 1 tonelada de residuos con la composición mostrada en el cuadro XII.

Los flujos de las diferentes fracciones en el escenario analizado se muestran en la figura 13.

Los datos de inventario considerados provienen de 
datos suministrados directamente por la empresa encargada de la gestión de los residuos, combinados con datos procedentes de la base de datos de White et al. (1995) y McDougall et al.(2001), ya que la tecnología utilizada en algunos procesos incluidos en Ecoinvent (2008) no eran adecuados para el caso de aplicación.

Los principales datos considerados en el estudio han sido los siguientes:

- Para la recogida, se considera un consumo de 19.751 de diésel por tonelada recogida.

- Para los diferentes transportes entre plantas y tratamientos, se ha considerado una distancia de $100 \mathrm{~km}$, con un camión de gran carga (40 t), al $70 \%$, y realizando un recorrido interurbano con un consumo de 1.921 de diésel por tonelada transportada.

- El porcentaje de impropios considerados para los procesos de reciclaje, son los mostrados en el cuadro XIII.

- El consumo eléctrico de la planta de separación de materiales es $2.45 \mathrm{kWh}$ por tonelada gestionada, siendo el mix eléctrico el mostrado en el cuadro XIV.

- Se considera la carga evitada debido al reciclaje de las fracciones separadas.

La figura 14 muestra los resultados de los indicadores ambientales relativos, obtenidos para cada categoría de impacto y distribuidos por las diferentes etapas del ciclo de vida consideradas.

En este caso de estudio, los impactos se deben a los consumos de combustible en las etapas de recogida y transporte a vertedero. Se observa que la carga evitada debido al bajo porcentaje de recuperación de las fracciones reciclables es incipiente, por lo que

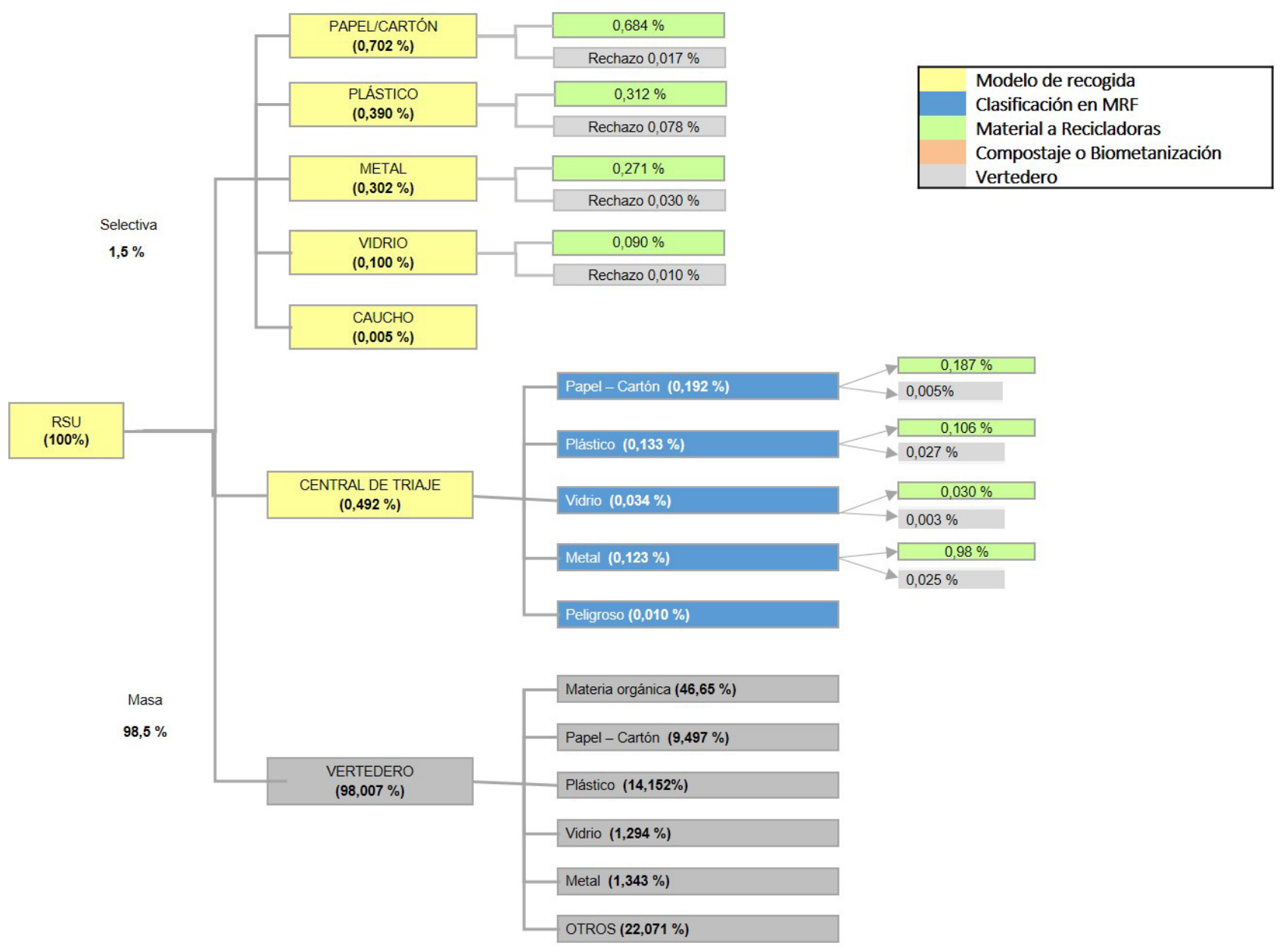

Fig. 13. Esquema del sistema de gestión de residuos considerado en el caso de estudio de Brasil 
CUADRO XIII. PORCENTAJE DE IMPROPIOS CONSIDERADOS PARA LOS PROCESOS DE RECICLAJE

\begin{tabular}{lll}
\hline Fracción & $\begin{array}{c}\text { Recogida selectiva } \\
(\%)\end{array}$ & $\begin{array}{c}\text { Recogida en masa } \\
(\%)\end{array}$ \\
\hline Metales Férricos & 90 & 80 \\
Metales no Férricos & 85 & 80 \\
Papel & 97.5 & 97.5 \\
Plástico & 80 & 80 \\
Vidrio & 90 & 90 \\
\hline
\end{tabular}

CUADRO XIV. MIX ELÉCTRICO CONSIDERADO EN
EL CASO DE ESTUDIO DE BRASIL,
PARA EL AÑO 2011

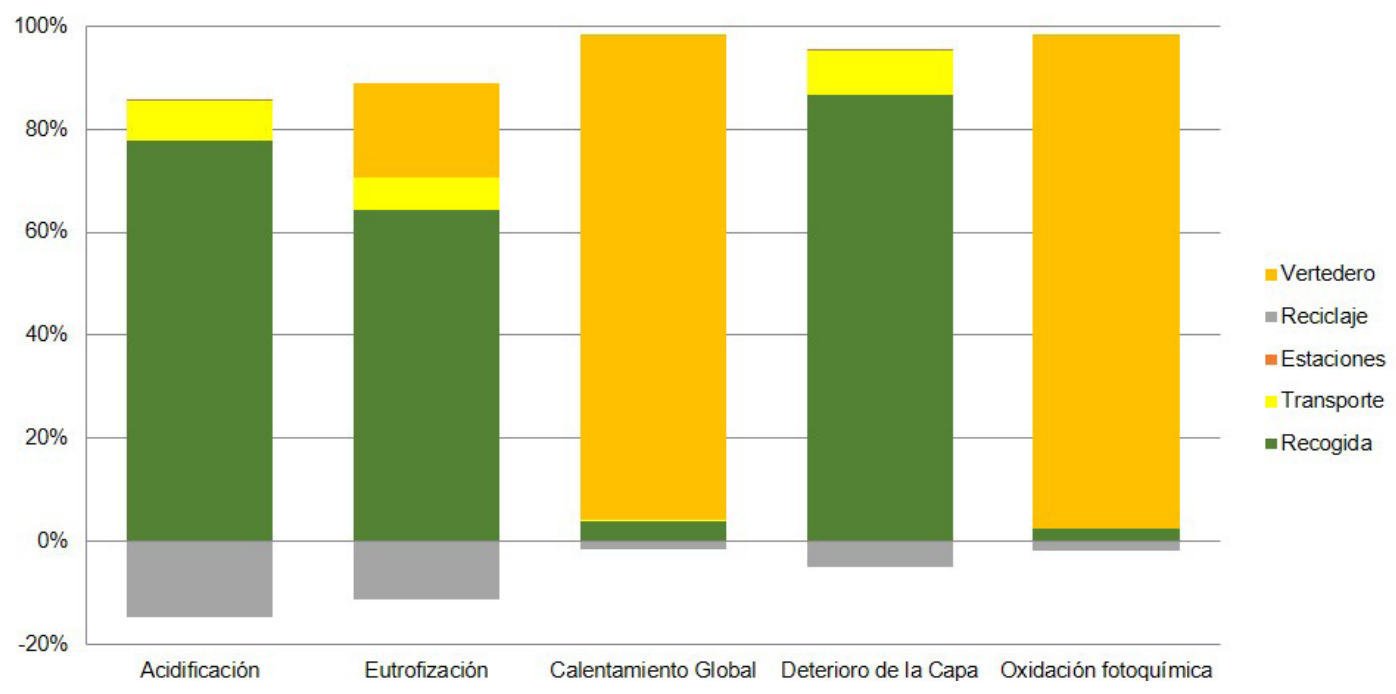

Fig. 14.Evaluación del impacto correspondiente al caso de aplicación de Brasil

las mejoras deberían ir enfocadas hacia optimizar el proceso de separación, incluido el de la materia orgánica (que actualmente se deposita toda en vertedero) de forma que podría incrementarse la carga evitada mientras se reduce el impacto en vertedero.

\section{Gestión de residuos domiciliarios (Colombia)}

El objetivo de este caso de estudio fue analizar el desempeño ambiental del ciclo de vida del actual sistema de gestión de los residuos domiciliarios producidos en la ciudad de Cartagena de Indias (Colombia), y compararlo con el de otras alternativas de gestión que podrían implementarse en un futuro.

Actualmente, no existe separación en origen, por lo que todos los residuos, orgánicos e inorgánicos, se reciben mezclados en bolsas y son recolectados por dos empresas que los transportan en camiones compactadores hasta el vertedero de Loma de los Cocos, donde son depositados sin ningún tipo de tratamiento o aprovechamiento.
La figura 15 muestra los tres escenarios a analizar:

- E1: sistema actual, previamente descrito.

- E2: aprovechamiento tanto los residuos orgánicos como inorgánicos a través de los procesos de compostaje y reciclaje.

- E3: aprovechamiento de los residuos tales como el vidrio, plástico, metales y papel-cartón a través de instalaciones de recuperación de materiales.

La unidad funcional corresponde a la gestión de los residuos sólidos generados en la ciudad de Cartagena de Indias durante el año 2007. Este valor se estimó en 285182 toneladas (Alcaldía de Cartagena y Universidad de Cartagena 2007), con la composición mostrada en el cuadro XV.

En el análisis del Inventario del Ciclo de Vida se cuantificaron los consumos de materias primas, energía, emisiones a la atmósfera, vertidos al agua y vertidos al suelo derivados de todos los procesos considerados dentro de los límites del sistema. 


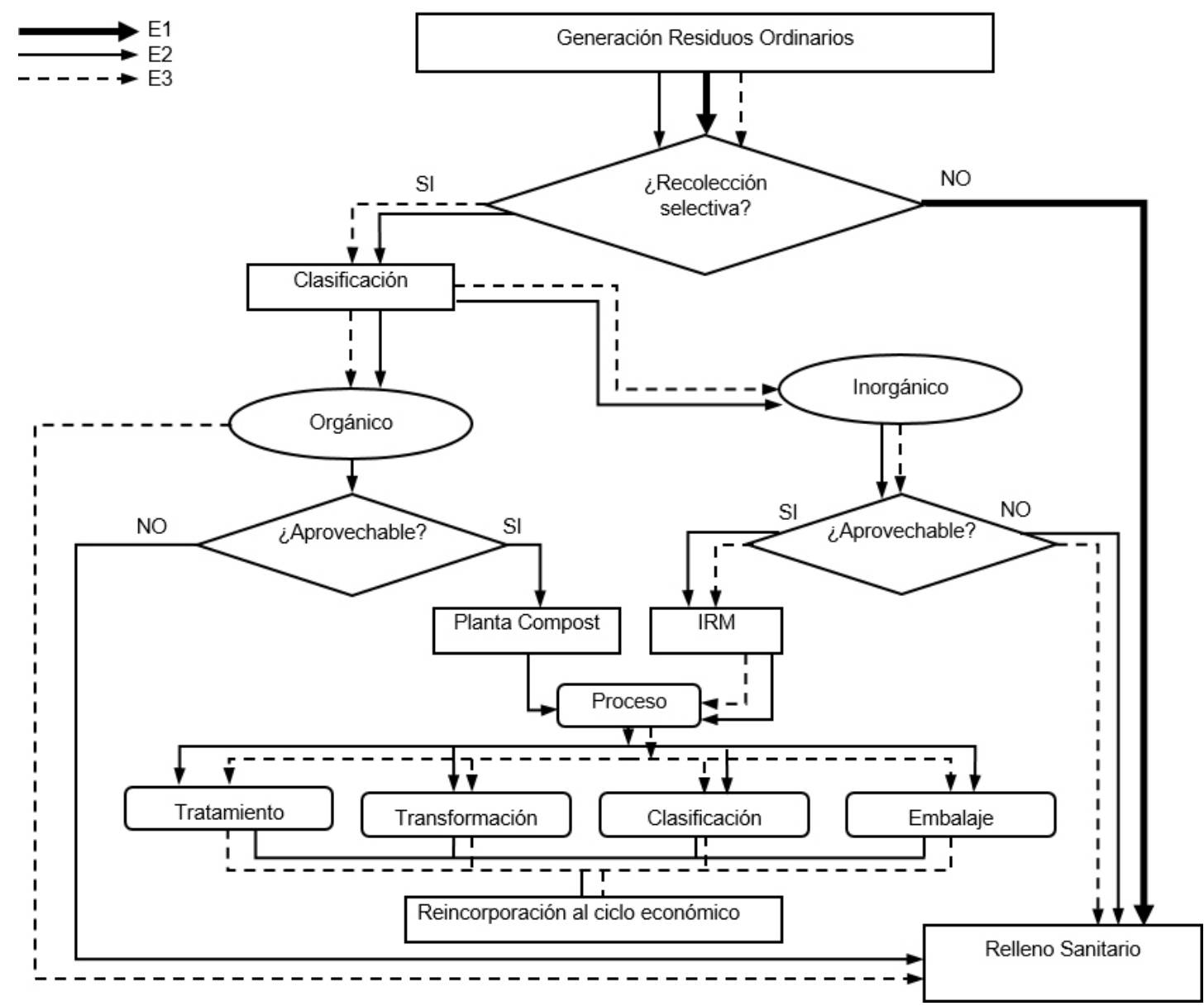

Fig. 15.Escenarios a evaluar y alcance de cada uno de ellos, en el caso de aplicación de Colombia (Quiñones et al. 2010)

CUADRO XV. COMPOSICIÓN DE LOS RESIDUOS SÓLIDOS PRODUCIDOS EN LA CIUDAD DE CARTAGENA

\begin{tabular}{lcccccc}
\hline Fracción & $\begin{array}{c}\text { Materia } \\
\text { orgánica }\end{array}$ & $\begin{array}{c}\text { Papel- } \\
\text { Cartón }\end{array}$ & Plástico & Vidrio & Metales & Otros \\
\hline$\%$ & 77.6 & 5.7 & 7.7 & 4.2 & 1 & 3.8 \\
\hline
\end{tabular}

Fuente: Alcaldía de Cartagena y Universidad de Cartagena 2007

Los datos de inventario considerados provienen de datos suministrados directamente por la empresa que supervisa el sistema de aseo en la ciudad de Cartagena, combinados con datos procedentes de la base de datos de la planta de compostaje de Torrelles de Llobregat (cuadro XVI). No se consideró la carga evitada que genera el aprovechamiento de los residuos.

En la figura 16 se presentan los resultados de la evaluación de impactos para los tres escenarios analizados en la ciudad de Cartagena para las categorías de impacto acidificación, calentamiento global, reducción de la capa de ozono y oxidación fotoquímica.

La disposición final de los residuos en vertedero genera contribuciones en todas las categorías de impacto, aunque predominantemente en agotamiento de la capa de ozono, seguido de calentamiento global, acidificación y oxidación fotoquímica. El reciclaje y el compostaje no generan mayores beneficios con 
CUADRO XVI. DATOS DE INVENTARIO CORRESPONDIENTES AL CASO DE APLICACIÓN DE COLOMBIA

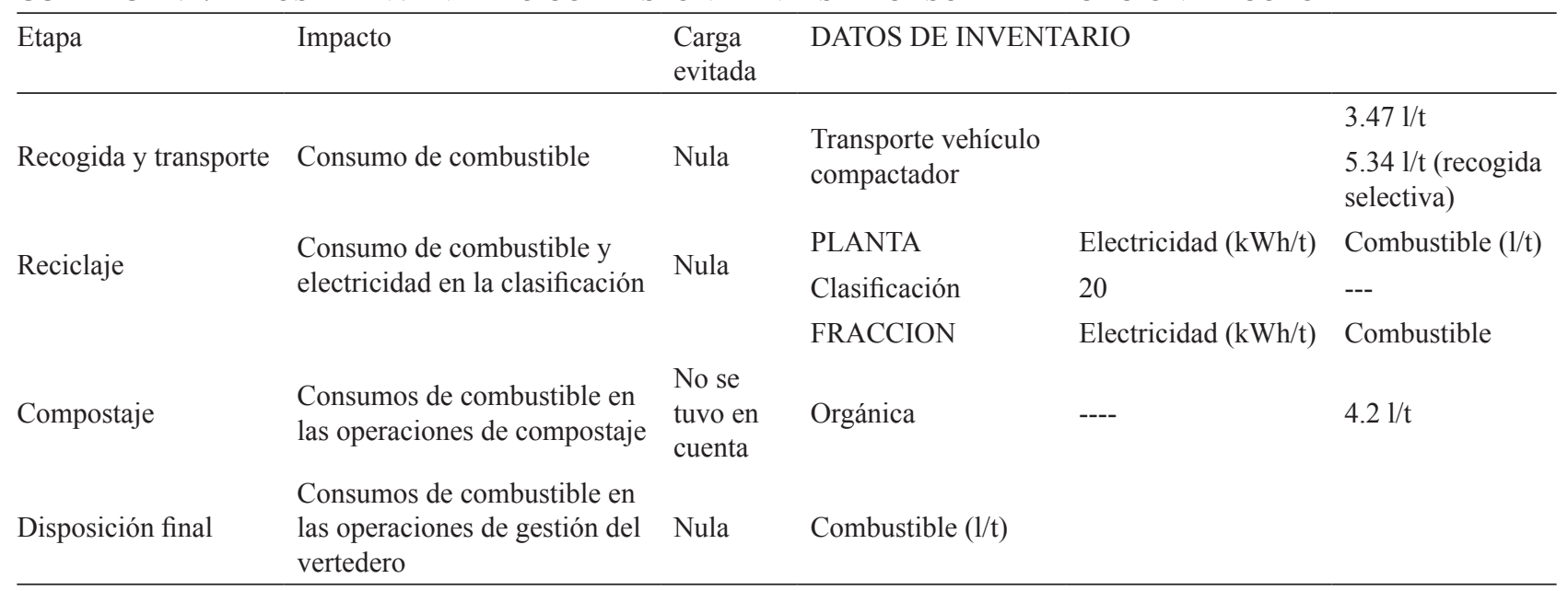

Fuente. Arzuzar-Centeno y Atencia-Herrera 2008

respecto a los impactos y esto se puede deber a los bajos porcentajes de material inorgánico aprovechable y al hecho de que no se han considerado los beneficios por cargas evitadas.

En la figura 17 se presenta la comparación de los tres escenarios evaluados. Se puede apreciar que el impacto por acidificación se da solamente en el escenario E2, que contempla el compostaje. Sin embargo, es el escenario que menor impacto genera en calentamiento global y disminución de la capa de ozono.

En la figura 17 se puede observar que el escenario con menor contribución al impacto es el E2, correspondiente al que involucra aprovechamiento de orgánicos e inorgánicos. Esto se debe a que la disminución en un $70 \%$ de las cantidades de residuos dispuestos (en comparación con la alternativa en la que no se aprovechan, E1), permite reducir en un $86 \%$ las emisiones de gases de efecto invernadero producidas en el relleno sanitario, con la ventaja adicional de una menor producción de lixiviados (86\%). Además, aumenta la vida útil del vertedero, por cuanto se disminuye la carga diaria de residuos (Quiñones et al. 2010).

Sin embargo, el escenario E3, a pesar de contemplar el aprovechamiento de inorgánicos, genera mayores impactos que el E1 (sin ningún tipo de aprovechamiento) y esto se debe a que la cantidad de inorgánicos es baja y se contempla la recogida selectiva que también genera impactos de relevancia.

\section{CONCLUSIONES}

Tal y como se ha visto es este artículo, los países iberoamericanos han iniciado en esta última década el uso de la metodología ACV para analizar el desempeño ambiental de sistemas de gestión de residuos. España, Portugal, Brasil, Chile, México y Colombia, por este orden, son los países que más publicaciones JCR tienen en esta área de investigación. Los residuos domiciliarios, seguidos de los de construcción y demolición e industriales-agroalimentarios, son las fracciones cuyos sistemas de gestión se han analizado desde una perspectiva de ciclo de vida mediante la metodología ACV.

En este artículo, además de analizar el estado del arte de la evaluación ambiental mediante técnicas de ACV en países iberoamericanos, se han presentado casos de estudio actuales correspondientes a España, México, Argentina, Brasil y Columbia, en los que se analiza, desde una perspectiva ambiental, la gestión de residuos domiciliarios, haciendo especial hincapié en la influencia que tiene la implementación de la recogida selectiva de fracciones reciclables; la gestión de los residuos de aparatos eléctricos y electrónico, haciendo especial énfasis en el beneficio ambiental que implica el reciclado de sus fracciones valorizables; y la gestión de los residuos de construcción y demolición, destacando la mejora ambiental que supone separar y valorizar determinadas fracciones.

Para cada uno de los casos de estudio, se han identificado las etapas críticas del sistema de gestión 


\section{Escenario 1}
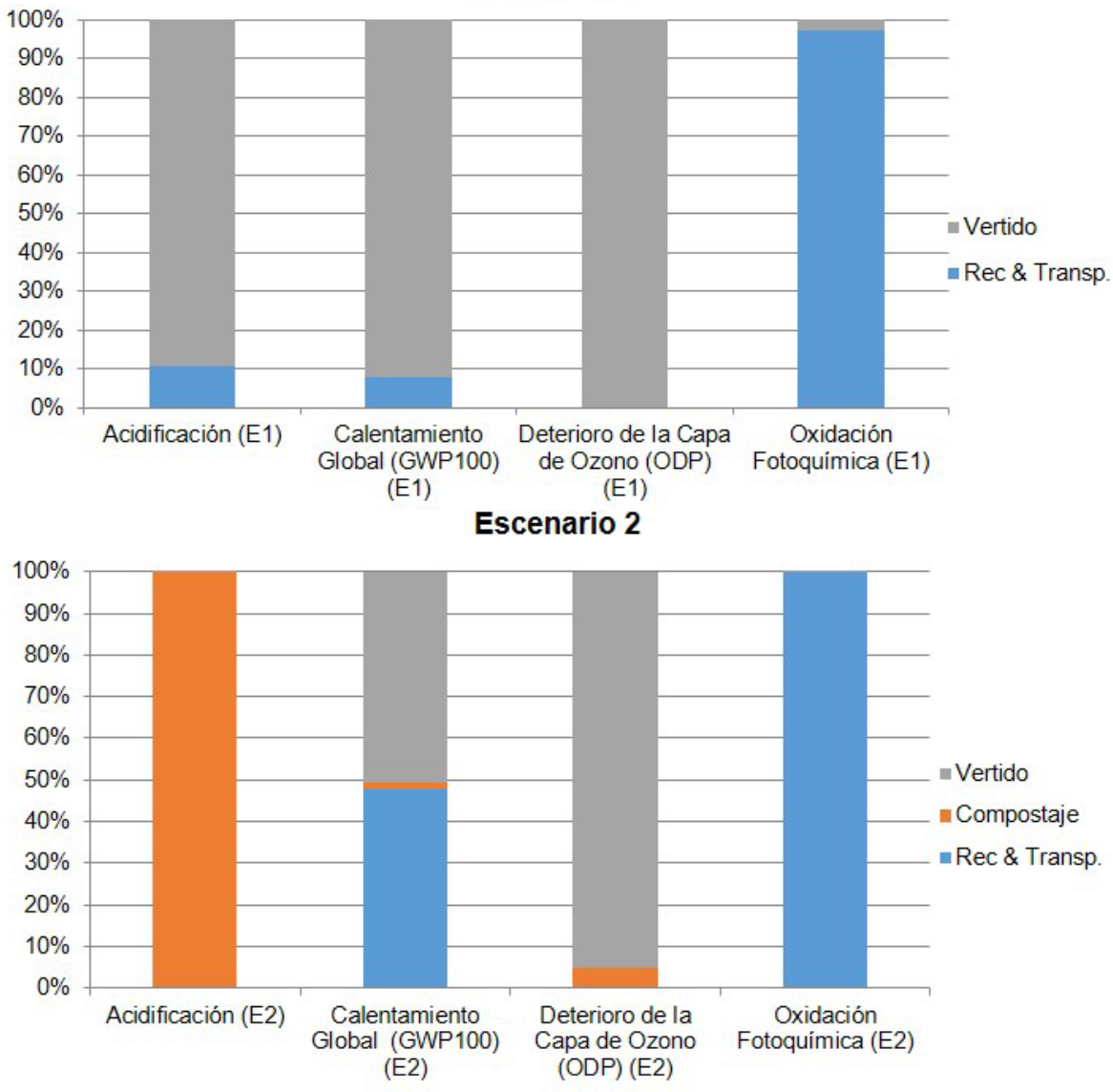

Escenario 3

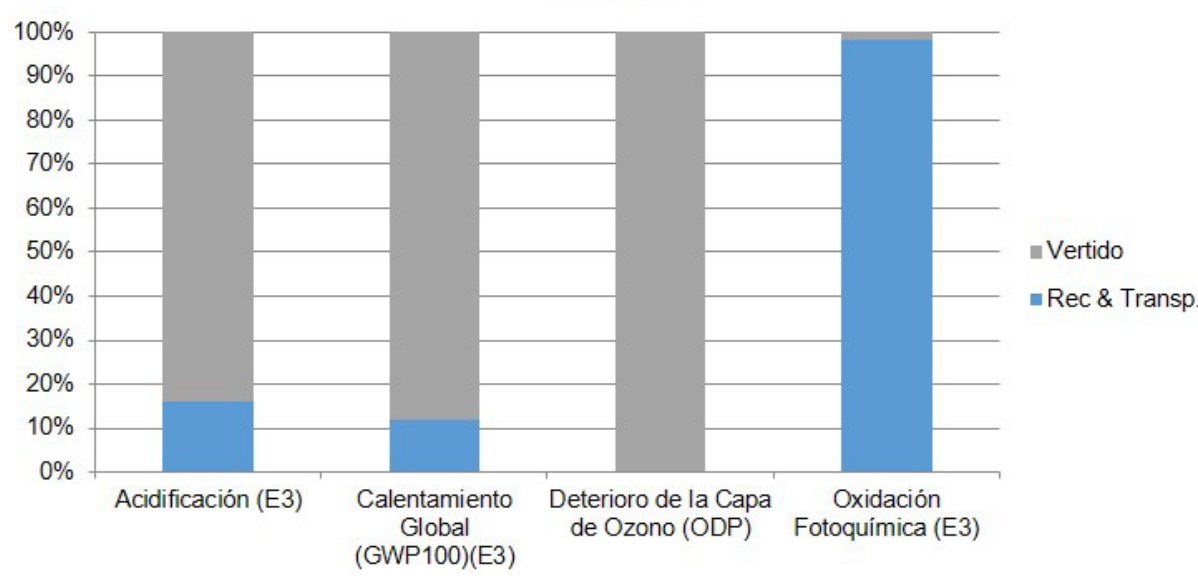

Fig. 16.Evaluación ambiental de los escenarios analizados en el caso de aplicación de Colombia 


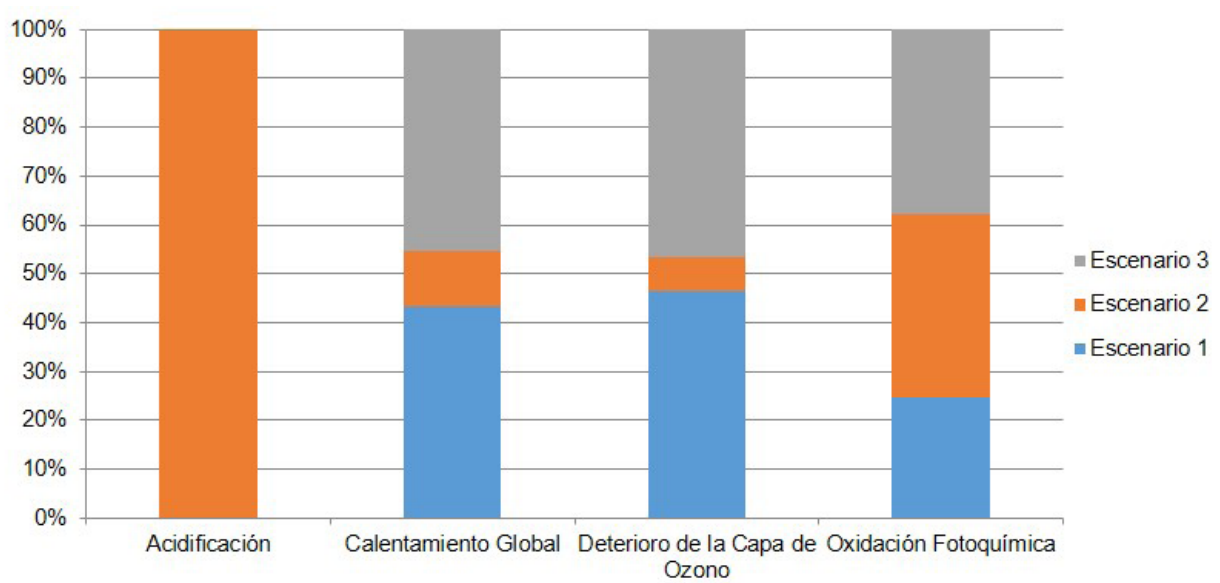

Fig. 17. Resultado final de la evaluación Ambiental de los tres escenarios, en el caso de estudio de Colombia

analizado, sirviendo como punto de partida para la selección e implantación de medidas que favorezcan la mejora continua de dichos procesos.

\section{AGRADECIMIENTOS}

Los autores agradecen la posibilidad de realizar este trabajo conjunto entre diferentes países iberoamericanos a CYTED (Proyecto 715RT0494).

\section{REFERENCIAS}

Alcaldía mayor de Cartagena de Indias y Universidad de Cartagena (2007). Plan de Gestión Integral de Residuos Sólidos de Cartagena de Indias (PGIRS). Informe Técnico Universidad de Cartagena. Cartagena de Indias. 220 páginas.

Aranda A., Ferreira G., Zambrana D., Zabalza I. y Llera E. (2013) Environmental-benefit analysis of two urban waste collection systems. Sci. Total Environ. 463-464, 72-77. DOI: 10.1016/j.scitotenv.2013.05.053

Arena U., Mastellone M.L. y Perugini F. (2003). Life cycle assessment of a plastic packaging recycling system. Int. J. Life Cycle Assess. 8 (2), 92-98.

DOI: $10.1007 / \mathrm{BF} 02978432$

Arzuzar L. y Atencia O. (2008). Aplicación del análisis del ciclo de vida (ACV) en la evaluación de alternativas para el manejo de residuos sólidos ordinarios en la ciudad de Cartagena de Indias. Tesis de Pregrado. Facultad de Ingeniería. Universidad de Cartagena.

Bezama A., Douglas C., Méndez J., Szarka N., Muñoz E., Navia R., Schock S., Konrad O. y Ulloa C. (2013) Life cycle comparison of waste-to-energy alternatives for municipal waste treatment in Chilean Patagonia. Waste Manage. Res. 31 (10 SUPPL.), 67-74.

DOI: $10.1177 / 0734242 X 13499810$

Bojacá C.R., Wyckhuys K.A.G.y Schrevens E. (2014) Life cycle assessment of Colombian greenhouse tomato production based on farmer-level survey data. J Clean Prod. 69, 26-33. DOI: 10.1016/j.scitotenv.2013.05.053

Bovea M.D., Ibanez-Fores V., Gallardo A. y ColomerMendoza F. (2010). Environmental assessment of alternative municipal solid waste management strategies. A Spanish case study. Waste Manage. 30, 2383-2395. http://dx.doi.org/10.1016/j.wasman.2010.03.001

Bovea, M. D., y Powell, J. C. (2006). Alternative scenarios to meet the demands of sustainable waste management. Journal of environmental management, 79(2), 115-132. http://dx.doi.org/10.1016/j.jenvman.2005.06.005

Cadena E., Colon J., Artola A., Sanchez A. y Font X. (2009). Environmental impact of two aerobic composting technologies using life cycle assessment. Int. J. Life Cycle Assess. 14, 401-410.

DOI: $10.1007 / \mathrm{s} 11367-009-0107-3$

CAMMESA (2013). Informe anual 2012. Compañía Administradora del Mercado Mayorista Eléctrico Sociedad Anónima. Argentina. http://portalweb.cammesa.com/ memnet1/Pages/descargas.aspx. Acceso: 09/08/14.

Ciroth A., Hageluken M., Sonnemann G.W., Castells F., Fleischer G. (2002)a. Geographical and technological differences in Life Cycle Inventories - shown by the use of process models for waste incinerators Part I. Technological and geographical differences. Int. J. Life Cycle Assess. 7, 295-300. DOI: 10.1007/BF02978891

Ciroth A., Hageluken M., Sonnemann G.W., Castells F. y Fleischer G. (2002)b. Geographical and technological differences in life cycle inventories - shown by the 
use of process models for waste incinerators part II: Technological and geographical differences. Int. J. Life Cycle Assess. 7, 363-368. DOI: 10.1007/BF02978685

Coelho A. y de Brito J. (2012). Influence of construction and demolition waste management on the environmental impact of buildings. Waste Manage. 32, 532-541. http://dx.doi.org/10.1016/j.wasman.2011.11.011

Colón J., Cadena E., Pognani M., Barrena R., Sanchez A. y Font X. y Artola A. (2012). Determination of the energy and environmental burdens associated with the biological treatment of source-separated municipal solid wastes. Energy Environ Sci. 5, 5731-5741. DOI: $10.1039 /$ C2EE01085B

Colón J., Martinez-Blanco J., Gabarrell X., Artola A., Sanchez A., Rieradevall J., Font X. (2010). Environmental assessment of home composting. Resour Conserv Recycl 54, 893-904.

DOI: $10.1016 /$ j. resconrec.2010.01.008

Ecoinvent (2008). The life cycle inventory data version 2. Swiss Centre for Life Cycle Inventories.

Ferrão P., Ribeiro P., Rodrigues J., Marques A., Preto M., Amaral M., Domingos T., Lopes A. y Costa E.I. (2014) Environmental, economic and social costs and benefits of a packaging waste management system: A Portuguese case study. Resour Conserv Recy. 85, 67-78. http://dx.doi.org/10.1016/j. resconrec.2013.10.020

Fonseca A.S., Nunes M.I., Matos M.A. y Gomes A.P. (2013) Environmental impacts of end-of-life vehicles' management: Recovery versus elimination. Int. J. Life Cycle Assess. 18, 1374-1385. DOI: 10.1007/s11367-013-0585-1

Frietas R.A., Menezes B., Helpa C.D.F. Soares S.R. (2012) The recycling of oyster shells: An environmental analysis using Life Cycle Assessment. J. Environ. Manage. 106, 102-109. DOI: 10.1016/j. jenvman.2012.04.017

Fullana I Palmer P., Puig R., Bala A., Baquero G., Riba J. y Raugei M. (2011) From life cycle assessment to life cycle management: A case study on industrial waste management policy making. J. Ind. Ecol. 15 (3), 458475. DOI: 10.1111/j.1530-9290.2011.00338.x

González-García S., Castanheira T.G., Dias A.C. y Arroja L. (2013) Using Life Cycle Assessment methodology to assess UHT milk production in Portugal. Sci. Total Environ. 442, 225-234.

DOI: 10.1016/j. jenvman.2012.04.017

Güereca L.P., Agell N., Gasso S. y Maria Baldasano J. (2007) Fuzzy approach to life cycle impact assessment - an application for biowaste management systems. Int. J. Life Cycle Assess. 12, 488-496.

DOI: $10.1065 /$ lca2006.10.276

Güereca L.P., Gassó S., Baldasano J.M. y Jiménez-Guerrero P. (2006) Life cycle assessment of two biowaste management systems for Barcelona, Spain. Resour.
Conserv. Recy. 49 (1), 32-48.

DOI: $10.1016 /$ j. resconrec.2006.03.009

Guinée J. (2002) Handbook on Life Cycle Assessment. An Operational Guide to the ISO Standards, Kluwer Academic Publishers.

Hospido A., Carballa M., Moreira M., Omil F., Lema J.M. y Feijoo G. (2010) Environmental assessment of anaerobically digested sludge reuse in agriculture: potential impacts of emerging micropollutants. Water Res. 44, 3225-3233. DOI: 10.1016/j. watres.2010.03.004

Hospido A., Moreira T., Martin M., Rigola M. y Feijoo G. (2005). Environmental evaluation of different treatment processes for sludge from urban wastewater treatments: anaerobic digestion versus thermal processes. Int. J. Life Cycle Assess. 10, 336-345.

DOI: $10.1065 /$ lca2005.05.210

Hou Q., Mao G., Zhao L., Du H. y Zuo J. (2015) Mapping the scientific research on life cycle assessment: a bibliometric analysis. Int. J. Life Cycle Assess. 20: 541-555. DOI: 10.1007/s11367-015-0846-2

IBGE (2012) Instituto Brasileiro de Geografia e Estatística. Contagem da População. http://www.ibge.gov.br/home/ estatistica/populacao/contagem2007/default.shtm

Iriarte A., Gabarrell X. y Rieradevall J. (2009). LCA of selective waste collection systems in dense urban areas. Waste Manage. 29, 903-914.

DOI: $10.1016 /$ j.wasman.2008.06.002

ISO 14040 (2006) Environmental Management. Life Cycle Assessment. Principles and Framework. ISO, Geneva. ISO 14044 (2006) Environmental Management. Life Cycle Assessment. Requirements and Guidelines. ISO, Geneva.

James K. (2012) An investigation of the relationship between recycling paper and card and greenhouse gas emissions from land use change Resour. Conserv. Recy. 67, 44-55. DOI: 10.1016/j.resconrec. 2012.07.003

Laurent A,. Bakas I., Clavreul J., Bernstad A., Niero M., Gentil E., Hauschild M.Z. y Christensen T.H. (2014) Review of LCA studies of solid waste management systems--part I: lessons learned and perspectives. Waste Manage. 34 (3):573-88 DOI: 10.1016/j.resconrec. 2012.07.003

Leme M.M.V., Rocha M.H., Lora E.E.S., Venturini O.J., Lopes B.M., Ferreira C.H. (2014) Techno-economic analysis and environmental impact assessment of energy recovery from Municipal Solid Waste (MSW) in Brazil. Resour. Conserv.Recy. 87, 8-20 DOI: 10.1016/j.resconrec.2014.03.003

Leme M.M.V., Rocha M.H., Lora E.E.S., Venturini O.J., Lopes, B.M. y Ferreira C.H. (2012) Environmental assessment of energy recovery technologies for the treatment and disposal of municipal solid waste using life cycle assessment (LCA): A case study of Brazil. 
Proceedings of the 25th International Conference on Efficiency, Cost, Optimization and Simulation of Energy Conversion Systems and Processes, ECOS 2012, 8, 62-70.

Margallo M., Aldaco R., Bala A., Fullana P. y Irabien A. (2010) Implementation of the selective collection in small villages of less than 50 inhabitants in Cantabria region (Spain): Preliminary viability study. Chem Eng Trans 21, 733-738. DOI: 10.3303/CET1021123

MARM (2008) (Ministerio de Medio Ambiente y Medio Rural y Marino) Plan Nacional de Residuos de Construcción y Demolición de España 2008-2015. Texto aprobado por Acuerdo de Consejo de Ministros de 26-12-2008. Boletín Oficial del Estado N. ${ }^{\circ} 49$ del 2602-2009, sección I, pp. 19893-20016

Martínez E., Nuñez Y. y Sobaberas E. (2013) End of life of buildings: Three alternatives, two scenarios. A case study. Int. J. Life Cycle Assess. 18 (5), 1082-1088. DOI: $10.1007 / \mathrm{s} 11367-013-0566-4$

McDougall F., White P., Franke M., Hindle P. (2001) Integrated Solid Waste Management: a Life Cycle Inventory, (second edition) Blackell Science Ltd.

Mendes M.R., Aramaki T. y Hanaki K. (2004) Comparison of the environmental impact of incineration and landfilling in São Paulo City as determined by LCA. Resour. Conserv. Recy. 41 (1), 47-63.

DOI: $10.1016 /$ j.resconrec.2003.08.003

Meneses M., Pasqualino J.C., Céspedes-Sánchez R. y Castells F. (2010) Alternatives for reducing the environmental impact of the main residue from a desalination plant. J. Ind. Ecol. 14 (3), 512-527.

DOI: $10.1111 / \mathrm{j} .1530-9290.2010 .00225 . x$

Mercante I. (2005) Impacto ambiental de los residuos de construcción y demolición. Alternativas de gestión, Tesis de Maestría en Ingeniería Ambiental. Universidad Nacional de Cuyo.

Mercante I.T., Bovea M.D., Ibáñez-Forés V. y Arena A.P. (2012) Life cycle assessment of construction and demolition waste management systems: A Spanish case study Int. J. Life Cycle Assess. 17 (2), 232-241.

DOI: $10.1007 / \mathrm{s} 11367-011-0350-2$

Montejo C., Tonini D., Márquez M.D.C. y Fruergaard T. (2013). Mechanical-biological treatment: Performance and potentials. An LCA of 8 MBT plants including waste characterization. J. Environ. Manage. 128, 661673. http://dx.doi.org/10.1016/j.jenvman.2013.05.063

Muñoz E. y Navia R. (2011) Life cycle assessment of solid waste management strategies in a chlor-alkali production facility. Waste Manage. Res. 29 (6), 634-643. DOI: $10.1177 / 0734242 X 10379492$

Muñoz I., Milà I Canals L. y Fernández-Alba A.R. (2010) Life cycle assessment of the average Spanish diet including human excretion Int. J. Life Cycle Assess.
15 (8), 794-805. DOI: 10.1007/s11367-010-0188-z

Muñoz I., Rieradevall J., Doménech X. y Milà L. (2004)

LCA application to integrated waste management planning in Gipuzkoa (Spain). Int. J. Life Cycle Assess. 9 (4), 272-280. DOI: 10.1007/BF02978603

Nóbrega C. C. (2003). Viabilidade Econômica, com Valorização Ambiental e Social, de Sistemas de Coleta Seletiva de Resíduos Sólidos Domiciliares- Estudo de Caso: João Pessoa/PB. Tese (Doutorado em Recursos Naturais) - Universidade Federal de Campina Grande, Campina Grande.

Ortiz O., Pasqualino J.C. y Castells F. (2010) Environmental performance of construction waste: Comparing three scenarios from a case study in Catalonia, Spain Waste Manage. 30 (4), 646-654.

DOI: 10.1016/j.resconrec.2003.08.003

Ortiz-Rodriguez O., Colodel C.M., Fischer M., Castells F., Sonnemann G. (2010) An application of Life Cycle Assessment (LCA) within the catalonian building sector: A case study Afinidad, 67 (548), 262-267.

Pires A. y Chang N.-B. y Martinho G. (2011) Reliabilitybased life cycle assessment for future solid waste management alternatives in Portugal Int. J. Life Cycle Assess, 16 (4), 316-337.

DOI: $10.1007 / \mathrm{s} 11367-011-0269-7$

Pires A., Martinho G. (2013) Life cycle assessment of a waste lubricant oil management system Int J Life Cycle Assess. 18 (1), 102-112.

DOI: $10.1007 / \mathrm{s} 11367-012-0455-2$

Puig R., Fullana-i-Palmer P., Baquero G., Riba J.-R. y Bala A. (2013) A cumulative energy demand indicator (CED), life cycle based, for industrial waste management decision making Waste Manage. 33 (12), 27892797. http://dx.doi.org/10.1016/j.wasman.2013.08.004

Quiñones Bolaños E., Mouthon Bello J. y Eljaiek Urzola M. (2010) Evaluación de Alternativas para el Manejo de Residuos Sólidos Ordinarios en la Ciudad de Cartagena de Indias Mediante la Metodología del Análisis del Ciclo de Vida. Proceedings del IV Simposio de Ingeniería de Residuos: Hacia la Sustentabilidad.

REE (2013). Informe anual 2012. Red Eléctrica Española, España.

Rieradevall J., Domenech X. y Fullana P. (1997). Application of life cycle assessment to landfilling. Int. J. Life Cycle Assess. 2, 141-144. DOI: 10.1007/BF02978806

Rivela B., Moreira M.T., Munoz I., Rieradevall J., Feijoo G. (2006). Life cycle assessment of wood wastes: a case study of ephemeral architecture. Sci. Total Environ. 357, 1-11. http://dx.doi.org/10.1016/j.scitotenv.2005.04.017

Rives J. y Rieradevall J., Gabarrell X. (2010). LCA comparison of container systems in municipal solid waste management. Waste Manage. 30, 949-957. 
DOI: 10.1016/j.resconrec.2003.08.003

Rodriguez-Iglesias J., Maranon E., Castrillon L., Riestra P. y Sastre H. (2003). Life cycle analysis of municipal solid waste management possibilities in Asturias, Spain. Waste Manage. Res. 21, 535-548. DOI: $10.1177 / 0734242 X 0302100606$

Romero-Hernández O., Romero Hernández S., Muñoz D., Detta-Silveira E., Palacios-Brun A. y Laguna, A. (2009) Environmental implications and market analysis of soft drink packaging systems in Mexico: a waste management approach. Int. J. Life Cycle Assess 14 (2), 07-113. DOI: 10.1007/s11367-008-0053-5

Rosen K. y Meier A. (2000) Energy use of US consumer electronics at the end of the 20th century. In: Proceedings of the Second International Conference on Energy Efficiency in Household Appliances. September 2000, Naples, Italy. LBLN-46212.

Rubin R.S., Castro M.A.S.D., Brandão D., Schalch V. y Ometto A.R. (2014) Utilization of Life Cycle Assessment methodology to compare two strategies for recovery of copper from printed circuit board scrap. J Clean. Prod. 64, 297-305.

DOI: 10.1016/j.resconrec.2003.08.003

Ruggieri L., Cadena E., Martinez-Blanco J., Gasol C.M., Rieradevall J., Gabarrell X., Gea T., Sort X., Sanchez A. (2009). Recovery of organic wastes in the Spanish wine industry. Technical, economic and environmental analyses of the composting process. J. Clean. Prod. 17, 830-838. DOI: 10.1016/j. jclepro.2008.12.005

Ruiz Peñalver S.M., Rodríguez Molina M. y Camacho Ballesta J.A. (2014) Direct and indirect generation of waste in the Spanish paper industry. Waste Manage. 34 (1), 3-11. http://dx.doi.org/10.1016/j.wasman.2013.09.014
SENER (2014) Secretaria de Energía. Estadísticas e indicadores del sector energético. Sector Eléctrico Nacional, Generación Bruta 2013-2014 <http:// egob2.energia.gob.mx/portal/ electricidad.html> 06/08/2014

SimaPro v8.0.1 (2013) PRé Consultants, B.V. Amersfoort, The Netherlands.

Soares S.R., Finotti A.R., Prudêncio da Silva, V. y Alvarenga R.A.F. (2013) Applications of life cycle assessment and cost analysis in health care waste management. Waste Manage. 33 (1), 175-183.

DOI: 10.1016/j.wasman.2012.09.021

Stichnothe H. y Azapagic A. (2009) Bioethanol from waste: Life cycle estimation of the greenhouse gas saving potential. Resour. Conserv. Recy. 53 (11), 624630. http://dx.doi.org/10.1016/j.resconrec.2009.04.012

Uggetti E., Ferrer I., Molist J. y Garcia J. (2011). Technical, economic and environmental assessment of sludge treatment wetlands. Water Res. 45, 573-582. http:// dx.doi.org/10.1016/j.watres.2010.09.019

Vinyes E., Oliver-Solà J., Ugaya C., Rieradevall J. y Gasol C.M. (2013) Application of LCSA to used cooking oil waste management. Int. J. Life Cycle Assess. 18 (2), 445-455. DOI: $10.1007 / \mathrm{s} 11367-012-0482-\mathrm{z}$

White P.R., Franke M. y Hindle P. (1995). Integrated Solid Waste Management: a Life Cycle Inventory. Blackie Academic \& Professional, London, UK.

Zanghelini G.M., Cherubini E., Orsi P. y Soares S.R. (2014) Waste management Life Cycle Assessment: The case of a reciprocating air compressor in Brazil. J Clean. Prod. 70, 164-174. White P.R., Franke M. y Hindle P. (1995). 